\title{
A proof of Finite Family Developments for Higher-Order Rewriting using a Prefix Property
}

\author{
H. J. Sander Bruggink \\ Department of Philosophy, Utrecht University \\ Email: bruggink@phil.uu.nl \\ Homepage: http://www .phil.uu.nl/ bruggink
}

\begin{abstract}
A prefix property is the property that, given a reduction, the ancestor of a prefix of the target is a prefix of the source. In this paper we prove a prefix property for the class of Higher-Order Rewriting Systems with patterns (HRSs), by reducing it to a similar prefix property of a $\lambda$-calculus with explicit substitutions. This prefix property is then used to prove that Higher-order Rewriting Systems enjoy Finite Family Developments. This property states, that reductions in which the creation depth of the redexes is bounded are finite, and is a useful tool to prove various properties of HRSs.
\end{abstract}

\section{Introduction}

Higher-order Rewriting Systems (HRSs), as introduced by Nipkow in 1991 [12,10], are a powerful tool to study the metatheory of declarative programming languages, like $\lambda$ Prolog and Haskell, on the one hand, and theorem provers and proof checkers, like Isabelle, on the other. Also, many (extensions of) $\lambda$-calculi can be encoded as instances of HRSs, so that results obtained for HRSs carry over to other interesting domains.

In this paper, we prove two properties of HRSs where left-hand sides of rule are restricted to be patterns. First we prove a prefix property, by reducing this property to a similar prefix property for a $\lambda$-calculus with explicit substitutions. The prefix property says that, given a step, the ancestor of a prefix of the target is a prefix of the source. Consider, as an example, the (first-order) rewriting system with the single rule

$$
\mathrm{f}(x) \rightarrow \mathrm{g}(\mathrm{f}(x), x)
$$

and the step $\mathrm{f}(\mathrm{h}(\mathrm{a})) \rightarrow \mathrm{g}(\mathrm{f}(\mathrm{h}(\mathrm{a})), \mathrm{h}(\mathrm{a}))$. Now, $p=\mathrm{g}(\mathrm{f}(\square), \mathrm{h}(\square))$ is a prefix of the target. Intuitively, its ancestor is $\mathrm{f}(\mathrm{h}(\square))$, because

$$
s=\mathrm{f}(\mathrm{h}(\square)) \rightarrow \mathrm{g}(\mathrm{f}(\mathrm{h}(\square)), \mathrm{h}(\square))=t
$$

and $p$ is contained in $t$. And indeed, $\mathrm{f}(\square)$ is a prefix of the source.

Many different prefix properties are possible: we can, e.g., vary in how the notions of prefix and ancestor are formalized, and we may impose additional 
conditions on the form of the prefixes. Prefix properties are already known for first-order TRSs [2,14] and (a labelling of) the $\lambda$-calculus with $\beta$-reductions [2], and have many applications, such as (head) needed reductions [14, Chap. 8] and normalization of outermost-fair reductions [14, Chap. 9]. A similar property is proved in Van Daalen's Square Brackets Lemma [15].

The second contribution is that we prove Finiteness of Family Developments (FFD) for HRS, by reducing this property to the prefix property described above. FFD states that each reduction, in which the "creation depth", or family, of function symbols is bounded, is finite. The intuition behind the notion of family is that in a step $C\left[l^{\sigma}\right] \rightarrow C\left[r^{\sigma}\right]$, the symbols of $r$ depend on the symbols of $l$, and therefore have a higher creation depth, while the symbols in $C$ and $\sigma$ do not take part in the step and have the same creation depth in both source and target. For example, consider the following infinite reduction, using the rewrite system above. We label the function symbols with their creation depth.

$$
f^{0}\left(a^{0}\right) \rightarrow g^{1}\left(f^{1}\left(a^{0}\right), a^{0}\right) \rightarrow g^{1}\left(g^{2}\left(f^{2}\left(a^{0}\right), a^{0}\right)\right) \rightarrow g^{1}\left(g^{2}\left(g^{3}\left(f^{3}\left(a^{0}\right), a^{0}\right), a^{0}\right)\right) \rightarrow \cdots
$$

Clearly, in this infinite reduction, the creation depth of the f's grows without bound. FFD states that restricting the creation depth to a finite number, yields finite reductions. FFD is a useful tool to prove various properties of rewrite systems, such as termination (e.g. termination of simply typed $\lambda$-calculus follows from FFD, see Appendix A), existence of standard reductions, etc.

Acknowledgements. I thank Vincent van Oostrom, Delia Kesner and the anonymous referees of the RTA 2006 conference for useful remarks on preliminary versions of this paper.

Note. This is an extended and more detailed version of [4]. Because this technical report includes additional definitions and lemmas, the numbering of examples, definitions, theorems and lemmas may differ in some places.

\section{Preliminaries}

We presuppose knowledge of the simply typed $\lambda$-calculus. Here we give a short overview of Higher-Order Rewrite Systems (HRSs) [10]. In particular, we consider HRSs as HORSs [16] with the simply typed $\lambda$-calculus as substitution calculus. We refer to [14, Sect. 11.2] for a good introduction.

Simple types are generated from a set of base types by the type constructor $\rightarrow$. Let $\Sigma$ be a signature of simply typed function symbols. We define a preterm to be a simply typed $\lambda$-terms over $\Sigma$. We want to consider $\beta \eta$-equivalence classes of preterms. Since it is well known that $\beta$-reduction, combined with restricted $\eta$ expansion ( $\bar{\eta}$-reduction), is confluent and terminating, we take $\beta \bar{\eta}$-normal forms as unique representatives of the $\beta \bar{\eta}$-equivalence classes. We define: a term is a preterm in $\beta \bar{\eta}$-normal form. In the following, $s, t$ will range over terms (and, whenever indicated, over preterms as well). 
A sequence $a_{1}, \ldots, a_{n}$ will sometimes be written as $\overline{a_{n}}$, or just $\bar{a}$ if the length is not important or clear from the context. Juxtaposition of two sequences denotes concatenation.

For terms or preterms $s, t_{1}, \ldots, t_{n}$, we write $s\left(t_{1}, \ldots, t_{n}\right)$ for $s t_{1} \cdots t_{n}$, or, in the case of terms, the $\beta$-normal form thereof. We also introduce the shorthand $\lambda \overline{x_{n}}$.s for $\lambda x_{1} \ldots \lambda x_{n} . s$. With $\mathrm{FV}(s)$ we denote the set of free variables of term or preterm $s$, and with $\operatorname{Sym}(s)$ the set of function symbols present in the term or preterm. If $\lambda \bar{x} \cdot a(\bar{s})$ is a term, then $a$ is called the head of that term ( $a$ is a function symbol or variable).

In the class of HRSs that we consider, the left-hand sides of rules are restricted to be local patterns. For patterns, unification is decidable and unique most general unifiers exist [11]. Local patterns, additionally, are linear (each free variable occurs at most once) and fully-extended (free variable have all bound variables in scope as argument). These extra requirements have a similar purpose as the requirement of left-linearity in first-order TRS: they keep matching local. To match a non-linear pattern, it is possible that subterms outside the pattern need to be checked for equality; to match a non fully extended pattern, it is possible that such a subterm must be checked for the non-occurrence of a variable. Because the notion of pattern depends on what the free variables are, we need to parametrize the notion with a context of variables, and obtain the following inductive definition:

Definition 2.1 (Pattern). Let $\bar{x}$ be a sequence of variables.

(i) A term $s$ is an $\bar{x}$-pattern if:

$-s=a\left(s_{1}, \ldots, s_{n}\right)$ and either $a \in \bar{x} \cup \Sigma$ and $s_{1}, \ldots, s_{n}$ are $\bar{x}$-patterns; or $s_{1}, \ldots, s_{n}$ is $\eta$-equivalent to a sequence of distinct variables from $\bar{x}$. $-s=\lambda y . s_{0}$ and $s_{0}$ is an $\bar{x} y$-pattern.

(ii) A term $s$ is linear outside $\bar{x}$, if each free variable which is not in $\bar{x}$, occurs in it at most once. A term $s$ is a fully extended $\bar{x}$-pattern, if, in the second case of the above definition, $s_{1}, \ldots, s_{n}={ }_{\eta} \bar{x}$. A term $s$ is a local $\bar{x}$-pattern, if $s$ is linear outside $\bar{x}$ and a fully extended $\bar{x}$-pattern.

Examples of local patterns are $\mathrm{f}(x), \mathrm{g}(\lambda x y . \mathrm{f}(z(x, y)))$ and $\mathrm{h}(\lambda x . z(x))$. Examples of non-local patterns are $\mathrm{g}(\lambda x y . \mathrm{f}(y))$ (not fully-extended) and $\mathrm{h}(\lambda x . z(x), \lambda x . z(x))$ (not linear). An example of a non-pattern is $\mathrm{g}(z(\mathrm{a}))$.

In the following, $p, q$ will range over patterns, and the word pattern (without the sequence of variables) will refer to a $\emptyset$-pattern.

Definition 2.2 (HRS). A rewrite rule (for a signature $\Sigma$ ) is a pair $\lambda \bar{x} . l_{0} \rightarrow$ $\lambda \bar{x} . r_{0}$ of closed $\Sigma$-terms of the same type, such that $l_{0}=f\left(s_{1}, \ldots, s_{n}\right)$ and $l_{0}$ is a local pattern not $\eta$-equivalent to a variable. An HRS is a tuple $\mathcal{H}=\langle\Sigma, R\rangle$, where $\Sigma$ is a signature and $R$ a set of rewrite rules for $\Sigma$.

The rewrite relation $\rightarrow_{\mathcal{H}}$ is defined as follows: $s \rightarrow_{\mathcal{H}} t$ if there exist a context $C$ such that $s={ }_{\beta} C[l]$ and $t={ }_{\beta} C[r]$, for some rule $l \rightarrow r \in R$.

For arbitrary rewrite system $\mathcal{R}$, we denote with $\rightarrow_{\mathcal{R}}$ the reflexive, transitive closure of $\rightarrow \mathcal{R}$. 
Note that there is no substitution in the definition of the rewrite relation, such as in first-order term rewriting systems (but see also Remark 2.4). The leading abstractions of the rules take the role of the substitution, as can be seen in the next example:

Example 2.3. Let the HRS Map, implementing the higher-order function map, be defined by:

$$
\begin{aligned}
\lambda z \cdot \operatorname{map}(\lambda x \cdot z(x), \text { nil }) & \rightarrow \lambda z \cdot \text { nil } \\
\lambda z u v \cdot \operatorname{map}(\lambda x \cdot z(x), \operatorname{cons}(u, v)) & \rightarrow \lambda z u v \cdot \operatorname{cons}(z(\mathrm{e}(u)), \operatorname{map}(\lambda x \cdot z(x), v))
\end{aligned}
$$

Here, cons and nil are the list constructors, viz. list composition and the empty list, respectively. The reason for the symbol e is to make the HRS non-collapsing (see Def. 2.5). A reduction of two $\mathcal{M}$ ap-steps is the following:

$$
\begin{aligned}
& \operatorname{map}(\lambda x . \mathrm{f}(x), \operatorname{cons}(\mathrm{a}, \mathrm{nil})) \\
& =\beta \quad(\lambda z u v \cdot \operatorname{map}(\lambda x . z(x), \operatorname{cons}(u, v)))\left(\lambda x^{\prime} . \mathrm{f}\left(x^{\prime}\right), \mathrm{a}, \mathrm{nil}\right) \\
& \rightarrow_{\mathcal{M} a p}(\overline{\lambda z u v \cdot \operatorname{cons}(z(\mathrm{e}(u)), \operatorname{map}(\lambda x . z(x), v))})\left(\lambda x^{\prime} . \mathrm{f}\left(x^{\prime}\right), \text { a, nil }\right) \\
& =\beta \quad \operatorname{cons}(\mathrm{f}(\mathrm{e}(\mathrm{a})), \operatorname{map}(\lambda x . \mathrm{f}(x), \mathrm{nil})) \\
& =\beta \quad \operatorname{cons}\left(\mathrm{f}(\mathrm{e}(\mathrm{a})),(\lambda z \cdot \operatorname{map}(\lambda x \cdot z(x), \operatorname{nil}))\left(\lambda x^{\prime} \cdot \mathrm{f}\left(x^{\prime}\right)\right)\right) \\
& \rightarrow \text { Map } \operatorname{cons}\left(\mathrm{f}(\mathrm{e}(\mathrm{a})),(\underline{\lambda z \cdot \mathrm{nil})})\left(\lambda x^{\prime} \cdot \mathrm{f}\left(x^{\prime}\right)\right)\right) \\
& =\beta \quad \operatorname{cons}(\mathrm{f}(\mathrm{e}(\mathrm{a})), \mathrm{nil})
\end{aligned}
$$

Note how the (underlined) left-hand sides are literally replaced by the (also underlined) right-hand sides.

In later examples, the leading abstractions of rewrite rules will be omitted; in other words, we will write $l \rightarrow r$ for $\lambda \bar{x} . l \rightarrow \lambda \bar{x} . r$.

Substitutions are maps from variables to terms. Application of a substitution $\sigma=\left[x_{1} \mapsto t_{1}, \ldots, x_{n} \mapsto t_{n}\right]$ to a term $s$ is defined as: $s^{\sigma}=\left(\lambda x_{1} \ldots x_{n} . s\right) t_{1} \ldots t_{n}$ (where this term is, as always, implicitly reduced to $\beta \bar{\eta}$-normal form). In the following, $\rho, \sigma, \tau, v$ will rangle over substitutions. The composition of substitions $\sigma$ and $\tau$ is denoted by $\sigma ; \tau$, where $s^{\sigma ; \tau}=\left(s^{\sigma}\right)^{\tau}$. A substitution is called linear, if each free variable occurs in its codomain at most once, i.e. if all terms of its codomain are linear and have mutually disjoint free variables. A (fully extended) $\bar{x}$-pattern substitution is a substitution of which the codomain consists of (fully extended) $\bar{x}$-patterns.

Remark 2.4. The rewrite relation of Def. 2.2 can alternatively, and more in the fashion of first-order TRSs, be defined in the following way:

$$
s \rightarrow \mathcal{H} t \quad \text { if } \quad s={ }_{\beta} C\left[l_{0}^{\sigma}\right] \text { and } t={ }_{\beta} C\left[r_{0}^{\sigma}\right]
$$

where $\lambda \bar{x} \cdot l_{0} \rightarrow \lambda \bar{x} \cdot r_{0} \in R$ and $\sigma$ is a substitution with $\operatorname{Dom}(\sigma)=\bar{x}$. This alternative definition, however, requires the notion of substitution to be defined, and therefore we prefer the other one. In the rest of the paper, we will sometimes implicitly switch definitions. 
Intuitively, a rewrite rule is collapsing, if it can bring context and subtitution, or different parts of the substitution, together, i.e. if, after the application of the rule, a function symbol of the context can be directly connected to a function symbol of the substitution. This can happen in two specific cases, which we will use as a definition:

Definition 2.5. A term $s$ is collapsing, if one of the following applies:

- (context-subst): $s=x\left(s_{1}, \ldots, s_{n}\right)$, where $x$ is a free variable; or

- (subst-subst): $s=C\left[x\left(s_{1}, \ldots, s_{n}\right)\right]$, and for some $k, s_{k}=\lambda \bar{z} \cdot y\left(t_{1}, \ldots, t_{m}\right)$, where $C$ is a context, $x$ is a free variable, and $y$ a free or bound variable.

$A$ rewrite rule $\lambda \bar{x} . l \rightarrow \lambda \bar{x} . r$ is collapsing, if $r$ is collapsing, and an HRS is collapsing, if at least one of its rules is.

Example 2.6.

- The rules $\lambda x . \mathrm{f}(x) \rightarrow \lambda x . x$ and $\lambda z \cdot \operatorname{mu}(\lambda x . z(x)) \rightarrow \lambda z . z(\operatorname{mu}(\lambda x . z(x)))$ are collapsing due to the (context-subst) condition. Suppose that $\mathrm{g}(\mathrm{a})$ is a redex, and consider the step $\mathrm{g}(\mathrm{f}(\mathrm{a})) \rightarrow \mathcal{H} \mathrm{g}(\mathrm{a})$. This step creates the redex $\mathrm{g}(\mathrm{a})$ without creating one of its function symbols.

- The rule $\lambda y z . \mathrm{g}(\lambda x . z(x), y) \rightarrow \lambda y z . \mathrm{f}(z(y))$ is collapsing due to the (substsubst) condition. Supppose that $\mathrm{f}(\mathrm{a})$ is a redex. Then the step $\mathrm{g}(\lambda x . \mathrm{f}(x), \mathrm{a}) \rightarrow$ $\mathrm{f}(\mathrm{a})$ creates the redex $\mathrm{f}(\mathrm{a})$ without creating one of its function symbols.

- The rule $\lambda y z \cdot \operatorname{app}(\operatorname{lam}(\lambda x . z(x)), y) \rightarrow \lambda y z . z(y)$ is collapsing due to both the (context-subst) and the (subst-subst) conditions.

An HRS $\mathcal{H}$ is terminating, if there are no infinite rewrite chains, i.e. if the inverse of its rewrite relation $\rightarrow \mathcal{H}$ is well-founded. Let $\subseteq$ be the subterm relation, i.e. the reflexive, transitive closure of the smallest relation $\subseteq_{1}$ such that $s_{1} \subseteq_{1} s_{1} s_{2}$, $s_{2} \subseteq_{1} s_{1} s_{2}, s \subseteq_{1} \lambda x . s$. We define: $\ll_{\mathcal{H}}=\leftarrow \mathcal{H} \cup \subseteq$.

Lemma 2.7. $\ll_{\mathcal{H}}$ is well-founded if and only if $\mathcal{H}$ is terminating.

Proof. Corresponds to [17, Lemma 7 (3)].

\section{Labelling HRSs with natural numbers}

Labelling rewriting systems is a well-known method to formalize the notion of redex family; see e.g. [8,9]. In this section, we develop a labelling, in the sense of $[18,14]$, for HRSs, analogous to the labelling for the $\lambda$-calculus used by Hyland [6] and Wadsworth [19]. Each function symbol is labelled by a natural number, representing the "creation depth" of the function symbol, and the rules are labelled such that every function symbol of the right-hand side is labelled with the largest label of the left-hand side plus one.

Definition 3.1 ( $\omega$-labelling).

(i) The $\omega$-labelling of a signature $\Sigma$ is defined as: $\Sigma^{\omega}=\left\{f^{\ell} \mid f \in \Sigma, \ell \in \mathbb{N}\right\}$. 
(ii) The family of a term $s$, denoted fam $(s)$, is the largest label of $s$, i.e.:

$$
\operatorname{fam}(s)=\max \left\{\ell \mid f^{\ell} \in \operatorname{Sym}(s)\right\}
$$

(iii) Let $s$ be a term, and $\ell \in \mathbb{N}$ a label. Then:

$$
\begin{aligned}
x\left(s_{1}, \ldots, s_{n}\right)^{\ell} & =x\left(s_{1}^{\ell}, \ldots, s_{n}^{\ell}\right) \\
f\left(s_{1}, \ldots, s_{n}\right)^{\ell} & =f^{\ell}\left(s_{1}^{\ell}, \ldots, s_{n}^{\ell}\right) \\
\left(\lambda x . s_{0}\right)^{\ell} & =\lambda x . s_{0}^{\ell}
\end{aligned}
$$

(iv) The projection operation $|\cdot|_{\omega}$ is the mapping from $\Sigma^{\omega}$ to $\Sigma$ given by $\left|f^{\ell}\right|_{\omega}=$ $f$. The mapping is homomorphically extended to terms.

(v) Let $\mathcal{H}=\langle\Sigma, R\rangle$. The $\omega$-labelled version of $\mathcal{H}$ is defined as: $\mathcal{H}^{\omega}=\left\langle\Sigma^{\omega}, R^{\omega}\right\rangle$, where $R^{\omega}$ consist of all rules $l^{\prime} \rightarrow r^{\left(\mathrm{fam}\left(l^{\prime}\right)+1\right)}$ such that $l \rightarrow r \in R$ and $\left|l^{\prime}\right|_{\omega}=l$.

The $\omega$-labelling only labels function symbols, not variables, abstractions or applications. The reason for this is that we want the $\omega$-labelling of an HRS to be an HRS itself (otherwise it would not be a labelling in the sense of $[18,14]$ ). Labelling variables is impossible, because $\alpha$-equivalent terms are identified. Labelling abstractions and applications is impossible because we have fixed the (unlabelled) simply typed $\lambda$-calculus as substitution calculus.

Example 3.2. The labelled HRS $\mathcal{M} a p^{\omega}$ consists, among others, of the rules:

$$
\begin{aligned}
\operatorname{map}^{0}\left(\lambda x . z(x), \text { nil }^{0}\right) & \rightarrow \text { nil }^{1} \\
\operatorname{map}^{1}\left(\lambda x . z(x), \text { nil }^{1}\right) & \rightarrow \text { nil }^{2} \\
\operatorname{map}^{0}\left(\lambda x . z(x), \operatorname{cons}^{0}(u, v)\right) & \rightarrow \operatorname{cons}^{1}\left(z\left(\mathrm{e}^{1}(u)\right), \operatorname{map}^{1}(\lambda x . z(x), v)\right) \\
\operatorname{map}^{0}\left(\lambda x . z(x), \operatorname{cons}^{1}(u, v)\right) & \rightarrow \operatorname{cons}^{2}\left(z\left(\mathrm{e}^{2}(u)\right), \operatorname{map}^{2}(\lambda x . z(x), v)\right)
\end{aligned}
$$

A labelled reduction corresponding to the reduction of Ex. 2.3 is the following:

$$
\begin{aligned}
& \operatorname{map}^{0}\left(\lambda x \cdot \mathrm{f}^{0}(x), \operatorname{cons}^{0}\left(\mathrm{a}^{0}, \text { nil }^{0}\right)\right) \\
& ={ }_{\beta} \quad\left(\lambda z u v \cdot \operatorname{map}^{0}\left(\lambda x . z(x), \operatorname{cons}^{0}(u, v)\right)\right)\left(\lambda x^{\prime} \cdot \mathrm{f}^{0}\left(x^{\prime}\right), \mathrm{a}^{0}, \text { nil }^{0}\right) \\
& \rightarrow \mathcal{M} a p\left(\overline{\left.\lambda z u v \cdot \operatorname{cons}^{1}\left(z\left(\mathrm{e}^{1}(u)\right), \operatorname{map}^{1}(\lambda x \cdot z(x), v)\right)\right)}\left(\lambda x^{\prime} \cdot \mathrm{f}^{0}\left(x^{\prime}\right), \mathrm{a}^{0}, \text { nil }^{0}\right)\right. \\
& ={ }_{\beta} \quad \operatorname{cons}^{1}\left(\mathrm{f}^{0}\left(\mathrm{e}^{1}\left(\mathrm{a}^{0}\right)\right), \operatorname{map}^{1}\left(\lambda x \cdot \mathrm{f}^{0}(x), \text { nil }^{0}\right)\right) \\
& =\beta \quad \operatorname{cons}^{1}\left(\mathrm{f}^{0}\left(\mathrm{e}^{1}\left(\mathrm{a}^{0}\right)\right),\left(\lambda z \cdot \operatorname{map}^{1}\left(\lambda x \cdot z(x), \mathrm{nil}^{0}\right)\right)\left(\lambda x^{\prime} \cdot \mathrm{f}^{0}\left(x^{\prime}\right)\right)\right) \\
& \rightarrow \text { Map } \operatorname{cons}^{1}\left(\mathrm{f}^{0}\left(\mathrm{e}^{1}\left(\mathrm{a}^{0}\right)\right),\left(\underline{\lambda z \cdot \mathrm{nil}^{2}}\right)\left(\lambda x^{\prime} \cdot \mathrm{f}^{0}\left(x^{\prime}\right)\right)\right) \\
& ={ }_{\beta} \quad \operatorname{cons}^{1}\left(f^{0}\left(e^{1}\left(a^{0}\right)\right), \text { nil }^{2}\right)
\end{aligned}
$$

Notice how only the labels of function symbols involved in the step (i.e. the underlined ones) are increased.

The following two lemmas provide a correspondence between labelled and unlabelled reductions:

Lemma 3.3. Let $\mathcal{H}$ be an HRS. $\mathcal{H}^{\omega}$ is orthogonal/collapsing/erasing, if and only if $\mathcal{H}$ is. 
Proof. By induction on the length we easily prove that $s^{\ell}$ is collapsing, if and only if $s$ is.

Lemma 3.4. Let $\mathcal{H}$ be an $H R S$.

(i) If $s \rightarrow \mathcal{H} t$, then, for each $s^{\prime}$ such that $\left|s^{\prime}\right|_{\omega}=s$, there is a $t^{\prime}$ such that $s^{\prime} \rightarrow \mathcal{H}^{\omega} t^{\prime}$ and $\left|t^{\prime}\right|_{\omega}=t$.

(ii) If $s \rightarrow \mathcal{H}^{\omega} t$, then $|s|_{\omega} \rightarrow \mathcal{H}|t|_{\omega}$.

Proof. Both items are easily proved by induction on the context of the step.

\section{The Prefix Property}

We call $p$ a prefix of term $t$, if it is a pattern, and there exists a substitution $\sigma$ such that $p^{\sigma}=t$. Given a step $s \rightarrow t$, a subterm $q$ of $s$ is the ancestor of a subterm $p$ of $t$, if the symbols of $t$ "trace to" the symbols of $s$. This notion is formalized here using labelling together with the rewrite relation: $q$ is an ancestor of $p$, if $\operatorname{fam}(p) \geq \operatorname{fam}(q)$ and $q \rightarrow \mathcal{H}^{\omega} p^{v}$. The substitution $v$ is necessary because $q$ might reduce to a "bigger" term than $p$; typically, $v$ has only function symbols which are also in $p$. Using these formalizations, we prove in this section the following theorem (proof begins on page 15).

Theorem 4.1 (Prefix Property). Let $\mathcal{H}^{\omega}$ be the $\omega$-labelling of a non-collapsing HRS, $s$ a term, $p$ a local $\bar{x}$-pattern and $\sigma$ a substitution. If $s \rightarrow \mathcal{H}^{\omega} p^{\sigma}$, then there exist a local $\bar{x}$-pattern $q$ and a substitution $\tau$, such that $s=q^{\tau}$, $\operatorname{fam}(p) \geq \operatorname{fam}(q)$, and either:

$$
\begin{aligned}
& -q \rightarrow \mathcal{H}^{\omega} p^{v}, \text { for some substitution } v \text { such that } v ; \tau=\sigma \text {; or } \\
& -q=p \text { and } \tau \rightarrow \mathcal{H}^{\omega} \sigma .
\end{aligned}
$$

The theorem states that, given a prefix of the target, its ancestor is a prefix of the source. There are two possibilities: either the prefix takes part in the step, or the step occurred fully in the substitution. Note that, in the first case, we do not only require that its ancestor is a prefix, but also that the suffix stays the same (except for duplicated subterms). In this regard, the lemma is stronger than e.g. the prefix property (for the $\lambda$-calculus) proved in [2, Prop. 7.4].

Example 4.2. Consider the following $\mathcal{M} a p^{\omega}$-step (see page 4):

$$
\mathrm{h}^{1}\left(\operatorname{map}^{3}\left(\lambda x \cdot \mathrm{f}^{2}(x), \operatorname{cons}^{2}\left(\mathrm{a}^{5}, \operatorname{nil}^{1}\right)\right) \rightarrow \mathrm{h}^{1}\left(\operatorname{cons}^{4}\left(\mathrm{f}^{2}\left(\mathrm{e}^{4}\left(\mathrm{a}^{5}\right)\right), \operatorname{map}^{4}\left(\lambda x . \mathrm{f}^{2}(x), \operatorname{nil}^{1}\right)\right)\right)\right.
$$

First, let the prefix $p=\mathrm{h}^{1}$ (cons $\left.{ }^{4}\left(\mathrm{f}^{2}\left(y_{1}\right), y_{2}\right)\right)$ of the target be given. The suffix is then given by $\sigma=\left[y_{1} \mapsto \mathrm{e}^{4}\left(\mathrm{a}^{5}\right), y_{2} \mapsto \operatorname{map}^{4}\left(\lambda x \cdot \mathrm{f}^{2}(x)\right.\right.$, nil $\left.\left.{ }^{1}\right)\right]$. Then:

$$
\begin{aligned}
q & =\mathrm{h}^{1}\left(\operatorname{map}^{3}\left(\lambda x . \mathrm{f}^{2}(x), \operatorname{cons}^{2}\left(y_{1}, z_{2}\right)\right)\right) \\
v & =\left[y_{1} \mapsto \mathrm{e}^{4}\left(z_{1}\right), y_{2} \mapsto \operatorname{map}^{4}\left(\lambda x \cdot \mathrm{f}(x), z_{2}\right)\right] \\
\tau & =\left[z_{1} \mapsto \mathrm{a}^{5}, z_{2} \mapsto \operatorname{nil}^{1}\right]
\end{aligned}
$$



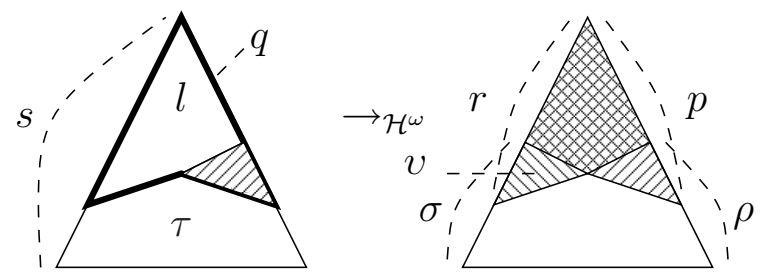

Fig. 1. The interesting case in the proof of the Prefix Property for HRSs

satisfy the conditions of the (trm) case. Second, let $p=\mathrm{h}(y)$ and $\sigma=[y \mapsto$ $\operatorname{cons}^{4}\left(f^{2}\left(\mathrm{e}^{4}\left(\mathrm{a}^{5}\right)\right), \operatorname{map}^{4}\left(\lambda x \cdot \mathrm{f}^{2}(x)\right.\right.$, nil $\left.\left.\left.\left.^{1}\right)\right)\right)\right]$. Then:

$$
q=\mathrm{h}^{1}(y) \quad \text { and } \quad \tau=\left[y \mapsto \operatorname{map}^{3}\left(\lambda x \cdot \mathrm{f}^{2}(x), \operatorname{cons}^{2}\left(\mathrm{a}^{5}, \mathrm{nil}^{1}\right)\right)\right]
$$

satisfy the conditions of the (sub) case.

The interesting case in the proof of the Prefix Property is the case that the step $s \rightarrow \mathcal{H}^{\omega} p^{\sigma}$ occurs at the head. In this case we have that $s=l^{\rho}$ and $p^{\sigma}=r^{\rho}$, for some rule $l \rightarrow r$ and substitution $\rho$. This situation is depicted in Fig. 1. We want to construct an ancestor $q$ that satisfies the (trm) case. It makes sense to try to do this by adding to the pattern $l$ the parts of $p$ that are not in $r$. However, due to the implicit $\beta$-conversions, these "parts of $p$ that are not in $r$ " are not easily obtained. The key observation is that the $\beta$-reduction from $p^{\sigma}$ to normal form is a variable renaming, because $p$ is a pattern and has only bound variables as arguments of free variables. The trick is to translate the prefix and suffix in such a way, that the variable renamings are already carried out (we need variable capturing, first-order substitutions for this, called graftings), trace the prefix back over the $\beta$-reduction from $r^{\rho}$ to normal form, and find the prefix's ancestor, which is a prefix of $r^{\rho}$. Now, we are dealing with terms that are exactly equal, instead of only equal up to $\beta$-equality, and now the problem can be solved by using first-order unification techniques.

The above proof technique suggests that we need to prove a prefix property for $\beta$-reductions in the $\lambda$-calculus. This is difficult, however, since the $\lambda$-calculus does not cope well with graftings, because of the global nature of substitution. For example, let $C=(\lambda x$. $\square)$ a, $D=\square$ and $s=x$. Then $C \rightarrow_{\beta} D$, and $C[s] \rightarrow_{\beta}$ a, because the $x$ in $s$ is captured by the abstraction in the context and substituted for. However, $D[s]=x$ and thus $C[s] \nrightarrow_{\beta} D[s]$. To tackle this problem, we use a $\lambda$-calculus with explicit substitutions, a variant of the $\lambda \mathrm{x}$-calculus, and prove a prefix property for it. Then, we simulate $\beta$-equality with this new calculus. In [5] a similar approach is taken w.r.t. higher-order unification.

\subsection{The Prefix Property of the $\lambda x$-Calculus}

We use a variant of the $\lambda \mathrm{x}$-calculus [3], with explicit renamings. The calculus has both object variables $(x, y, z)$ and metavariables $(X, Y, Z)$. In the following, 
we will refer to it simply by $\lambda$ x-calculus. The terms of the $\lambda$ x-calculus over some signature $\Sigma$ are first-order terms given by the following grammar:

$$
\Lambda_{\mathrm{x}}:=x|X| f\left|\lambda x \cdot \Lambda_{\mathrm{x}}\right| \Lambda_{\mathrm{x}} \Lambda_{\mathrm{x}} \mid \Lambda_{\mathrm{x}}\left\{x \backslash \Lambda_{\mathrm{x}}\right\}
$$

where $f \in \Sigma$ and the object variables are considered as constants or names. $M, N$ will range over $\lambda$ x-terms. Terms of the form $M\{x \backslash N\}$ will be called explicit substitutions, and the $\{x \backslash N\}$ part of an explicit substitution is called a closure. With $\operatorname{MV}(M)$ we will denote the set of metavariables of $M$, and with $\operatorname{Sym}(M)$ the set of function symbols of $M$. The reduction rules of the $\lambda$ x-calculus are:

$$
\begin{array}{ll} 
& (\lambda x \cdot M) N \rightarrow \mathrm{B} M\{x \backslash N\} \\
x\{x \backslash N\} \rightarrow_{\mathrm{x}} N & (\lambda x \cdot M)\{x \backslash N\} \rightarrow_{\mathrm{x}} \lambda x \cdot M \\
y\{x \backslash N\} \rightarrow_{\mathrm{x}} y & (\lambda y \cdot M)\{x \backslash N\} \rightarrow_{\mathrm{x}} \lambda z \cdot M\{y \backslash z\}\{x \backslash N\} \\
f\{x \backslash N\} \rightarrow_{\mathrm{x}} f & \left(M_{1} M_{2}\right)\{x \backslash N\} \rightarrow_{\mathrm{x}} M_{1}\{x \backslash N\} M_{2}\{x \backslash N\}
\end{array}
$$

where $x \neq y$ and $z$ is a fresh object variable. The subcalculus $\mathrm{x}$ consists of all rules except the B-rule. The reduction relations $\rightarrow_{\mathrm{Bx}}$ and $\rightarrow_{\mathrm{x}}$ are the contextual closures of the above steps. Note that there is no reduction rule for terms of the form $X\{x \backslash N\}$, where $X$ is a metavariable, and thus x-normal forms are characterized by the fact that sequences of closures are only applied to metavariables.

A $\lambda \mathrm{x}$-term is called passive if no metavariable $X$ occurs in a subterm of the form $X \bar{\mu}\left(M_{1}, \ldots, M_{n}\right)$, where $\bar{\mu}$ is a sequence of closures; it is called linear, if every metavariable occurs in it at most once. In the following $P, Q$ will range over linear, passive $\lambda \mathrm{x}$-terms.

Remark 4.3. It is well-known that the $\lambda$ x-calculus is not confluent on terms containing metavariables, as is witnessed by the following counterexample:

$$
\begin{array}{ll}
(\lambda x .(\lambda y . Z) Y) X & (\lambda x .(\lambda y \cdot Z) Y) X \\
\rightarrow_{\mathrm{Bx}}\left(\lambda y^{\prime} . Z\left\{y \backslash y^{\prime}\right\}\{x \backslash X\}\right) Y\{x \backslash X\} & \rightarrow_{\mathrm{Bx}}(\lambda x . Z\{y \backslash Y\}) X \\
\rightarrow_{\mathrm{Bx}} Z\left\{y \backslash y^{\prime}\right\}\{x \backslash X\}\left\{y^{\prime} \backslash Y\{x \backslash X\}\right\} & \rightarrow_{\mathrm{Bx}} Z\{y \backslash Y\}\{x \backslash X\}
\end{array}
$$

At first sight, non-confluence seems problematic, because we're trying to use the $\lambda \mathrm{x}$-calculus to simulate the (confluent) $\lambda$-calculus. However, the translation to $\lambda$-calculus (see page 13) will remove all closures, and will project normal forms of the same $\lambda$ x-term to the same $\lambda$-term (modulo $\alpha$-equivalence).

A grafting is a mapping from metavariables to $\lambda \mathrm{x}$-terms. The greek lowercase letters $\zeta, \eta, \theta, \kappa$ will range over graftings. Applying a grafting $\zeta$ to a term $M$, written $M[\zeta]$, is defined exactly as first order substitution, i.e.:

$$
\begin{aligned}
x[\zeta] & =x & (\lambda x \cdot M)[\zeta] & =\lambda x \cdot M[\zeta] \\
X[\zeta] & =\zeta^{\prime}(X) & \left(M_{1} M_{2}\right)[\zeta] & =M_{1}[\zeta] M_{2}[\zeta] \\
f[\zeta] & =f & (M\{x \backslash N\})[\zeta] & =M[\zeta]\{x \backslash N[\zeta]\}
\end{aligned}
$$

where $\zeta^{\prime}(X)=\zeta(X)$, if $X \in \operatorname{Dom}(\zeta)$, and $\zeta^{\prime}(X)=X$, otherwise. A grafting is called linear, if every metavariable occurs in its codomain only once, i.e. its 
codomain consists of linear $\lambda \mathrm{x}$-terms with mutually disjoint metavariables. A grafting is called passive, if all the terms of its codomain are passive.

Because $\lambda \mathrm{x}$-terms are first-order terms, unification is decidable. In the proof of the Prefix Property, we need the following property: if two $\lambda x$-terms are unifiable, there exists a most general unifier (mgu). In fact, if we assume the unifiable terms to be linear and passive, then the mgu applied to one of the terms is a linear, passive $\lambda \mathrm{x}$-term again:

Lemma 4.4. Let $M, N$ be linear $\lambda \mathrm{x}$-terms, where $\operatorname{MV}(M) \cap \operatorname{MV}(N)=\emptyset$, and let $\zeta, \eta$ be graftings such that $M[\zeta]=N[\eta]$. There exist graftings $\zeta_{0}, \eta_{0}, \kappa$ such that $M\left[\zeta_{0}\right]=N\left[\eta_{0}\right], \zeta_{0} ; \kappa=\zeta, \eta_{0} ; \kappa=\eta, \operatorname{Sym}\left(\zeta_{0}\right) \subseteq \operatorname{Sym}(N), \operatorname{Sym}\left(\eta_{0}\right) \subseteq \operatorname{Sym}(M)$. Moreover, if $M(N)$ is passive, then $\eta_{0}\left(\zeta_{0}\right)$ consists of passive $\lambda \mathrm{x}$-terms.

Proof. Since we consider $\lambda \mathrm{x}$-terms here as first-order terms, the (first part) of the lemma is essentially an instance of first-order unification.

By assumption, $M$ and $N$ are unified by $\langle\mu, \nu\rangle$ (the assumption that the metavariables are disjoint allows us to consider $\zeta$ and $\eta$ together as a unifier), and thus have a most general unifier (mgu). We take $\left\langle\zeta_{0}, \eta_{0}\right\rangle$ to be this mgu. The desired $\kappa$ exists because any unifier is an instance of an mgu.

Assume $f \in \operatorname{Sym}\left(\eta_{0}\right)$, but $f \notin \operatorname{Sym}(M)$. Then it must be the case that $f \in \operatorname{Sym}\left(\zeta_{0}\right)$. Since all the subterms of $f$ must be in the graftings too, there exist graftings $\zeta_{0}^{\prime}, \eta_{0}^{\prime}, \kappa^{\prime}$ such that $\zeta_{0}^{\prime} ; \kappa=\zeta_{0}$ and $\eta_{0}^{\prime} ; \kappa=\eta_{0}$. It follows by linearity that $\left\langle\zeta_{0}^{\prime}, \eta_{0}^{\prime}\right\rangle$ is a unifier, contradicting the assumption. For the assumption that $f \in \operatorname{Sym}\left(\zeta_{0}\right)$, but $f \notin \operatorname{Sym}(N)$, a contradiction is derived through a symmetrical argument.

For the second part of the lemma, suppose $M$ is passive, but $\eta_{0}(X)$ is not. Then $N\left[\eta_{0}\right]$ is not passive, and contains a subterm of the form $N_{0}=$ $X \bar{\mu}\left(N_{1}, \ldots, N_{n}\right)$. Because $M\left[\zeta_{0}\right]=N\left[\eta_{0}\right]$, so does $M\left[\zeta_{0}\right]$. There are two possibilities: if the root application of $N_{0}$ is in $M$, then $M$ is not passive, contradicting the assumption; otherwise, $N_{0}$ must be a subterm of $\zeta_{0}(Y)$, for some $Y \in \operatorname{Dom}\left(\mu_{0}\right)$, but then $\left\langle\zeta_{0}, \eta_{0}\right\rangle$ is not a mgu. Again, a symmetrical arguments yields the desired result if $N$ is passive, but $\mu_{0}(X)$ is not.

Example 4.5. Let:

$$
\begin{aligned}
M & =\lambda x \cdot \mathrm{g}\left(\mathrm{f}_{1}\left(X_{1}\right), X_{2}\right) & N & =\lambda x \cdot \mathrm{g}\left(Y_{1}, \mathrm{f}_{2}\left(Y_{2}\right)\right) \\
\zeta & =\left[X_{1} \mapsto \mathrm{a}, X_{2} \mapsto \mathrm{f}_{2}(\mathrm{a})\right] & \eta & =\left[Y_{1} \mapsto \mathrm{f}_{1}(\mathrm{a}), Y_{2} \mapsto \mathrm{a}\right]
\end{aligned}
$$

Then $M[\zeta]=\lambda x \cdot g\left(\mathrm{f}_{1}(\mathrm{a}), \mathrm{f}_{2}(\mathrm{a})\right)=N[\eta]$. We take $\zeta_{0}=\left[X_{2} \mapsto \mathrm{f}_{2}\left(Z_{1}\right)\right], \eta_{0}=\left[Y_{1} \mapsto\right.$ $\left.\mathrm{f}_{1}\left(Z_{2}\right)\right]$ and $\kappa=\left[Z_{i} \mapsto \mathrm{a}\right]$ to satisfy the conditions of the lemma.

In the next theorem, we prove the Prefix Property for the $\lambda \mathrm{x}$-calculus. $P$ is a prefix of a $\lambda \mathrm{x}$-term $M$, if it is a linear, passive $\lambda \mathrm{x}$-term, and there exists a grafing $\zeta$ such that $P[\zeta]=M$. The notion of ancestor is again formalized using labelling and the rewrite relation; however, because we do not count creation depth in Bx-reductions, now the labels, or more generally, the function symbols of the prefix must be the same as those of its ancestor. Just like in Theorem 4.1, a prefix can either take part in the step, or not, resulting in two cases. Item (ii) is the extension of the Prefix Property to Bx-reductions. 
Theorem 4.6 ( $\lambda \mathrm{x}$-Prefix Property). Let $M$ be a closed $\lambda \mathrm{x}$-term, $P$ a linear, passive $\lambda \mathrm{x}$-term and $\zeta$ a grafting.

(i) If $M \rightarrow_{\mathrm{Bx}} P[\zeta]$, then there exist a linear, passive $\lambda \mathrm{x}$-term $Q$ and a grafting $\eta$ such that $M=Q[\eta]$, Sym $(Q)=\operatorname{Sym}(P)$ and either:

$-Q \rightarrow_{\mathrm{Bx}} P[\kappa]$ where $\kappa$ is some grafting such that $\kappa ; \eta=\zeta$; or $\quad$ (trm)

$-Q=P$ and $\eta \rightarrow_{\mathrm{x}} \zeta$.

(ii) If $M \rightarrow \mathrm{Bx} P[\zeta]$, then there exist a linear, passive $\lambda \mathrm{x}$-term $Q$ and a grafting $\eta$ such that: $M=Q[\eta], \operatorname{Sym}(Q)=\operatorname{Sym}(P), Q \rightarrow_{\mathrm{Bx}} P[\kappa]$ where $\kappa$ is some grafting such that $\kappa ; \eta \rightarrow_{\mathrm{Bx}} \zeta$.

Proof. (i) By induction on the context of the step $M \rightarrow_{\mathrm{Bx}} P[\zeta]$. In this proof, let $\mathrm{id}_{\zeta}$ be the identity grafting on the domain of a grafting $\zeta$, i.e. $\operatorname{id}_{\zeta}:=[X \mapsto$ $X \mid X \in \operatorname{Dom}(\zeta)]$.

If $P=X$, then we take $Q=X$ and $\eta=\zeta[X \mapsto M]$, satisfying the (sub) case of the lemma. If the step does not occur at the head of the term, then the lemma follows simply from the induction hypothesis. Otherwise we look at the reduction rule which was applied (at the head).

The interesting case is when a closure is distributed over an application: i.e. $M=\left(M_{1} M_{2}\right)\{x \backslash N\} \rightarrow_{\mathrm{x}}\left(M_{1}\{x \backslash N\}\right)\left(M_{2}\{x \backslash N\}\right)=P[\zeta]$. Since $P$ is not a metavariable, $P=P_{1} P_{2}$, where $P_{1}[\zeta]=M_{1}\{x \backslash N\}$ and $P_{2}[\zeta]=M_{2}\{x \backslash N\}$. For $P_{2}$, there are two possible cases:

(a) If $P_{2}=X$, for some metavariable $X$, then let $\kappa^{\prime}=\mathrm{id}_{\zeta} ;[X \mapsto Y\{x \backslash Z\}]$ and $\zeta^{\prime}=\zeta\left[X \mapsto X, Y \mapsto M_{2}, Z \mapsto N\right]$, where $Y, Z$ are fresh metavariables.

(b) Otherwise, let $\kappa^{\prime}=\mathrm{id}_{\zeta}$ and $\zeta^{\prime}=\zeta$.

In both cases, $P_{2}\left[\kappa^{\prime}\right]=Q_{2}\left\{x \backslash R_{2}\right\}$, for some passive $\lambda$ x-terms $Q_{2}$ and $R_{2}$, and $\kappa^{\prime} ; \zeta^{\prime}=\zeta$.

Since $P$ is passive, we know that $P_{1}$ is not of the form $X \bar{\mu}$, where $\bar{\sigma}$ is a list of substitutions. In particular, $\bar{\mu}$ is not empty. Thus, $P_{1}=Q_{1}\left\{x \backslash R_{1}\right\}$. Because $P$ is linear, and the $\kappa^{\prime}(X)=X$ for $X \in \operatorname{MV}\left(P_{1}\right), P_{1}\left[\kappa^{\prime}\right]=P_{1}$. Also, because in case (a), $Y$ and $Z$ are fresh metavariables, and in case (b), $\zeta^{\prime}=\zeta$, it holds that $P_{1}\left[\zeta^{\prime}\right]=P_{1}[\zeta]$.

Because $P$ is linear, and $R_{1}$ and $R_{2}$ are different subterms of $P, R_{1}$ and $R_{2}$ are linear and have no metavariables in common. Since $R_{1}\left[\zeta^{\prime}\right]=N=R_{2}\left[\zeta^{\prime}\right]$, we can apply Lemma 4.4, and obtain graftings $\zeta_{1}, \zeta_{2}, \eta$ such that $R_{1}\left[\zeta_{1}\right]=R_{2}\left[\zeta_{2}\right]$, $\zeta_{1} ; \eta=\zeta^{\prime}, \zeta_{2} ; \eta=\zeta^{\prime}, \operatorname{Sym}\left(\zeta_{1}\right) \subseteq \operatorname{Sym}\left(R_{2}\right)$ and $\operatorname{Sym}\left(\zeta_{2}\right) \subseteq \operatorname{Sym}\left(R_{1}\right)$.

Let $R=R_{1}\left[\zeta_{1}\right]\left(=R_{2}\left[\zeta_{2}\right]\right)$. Because $\operatorname{Sym}\left(\zeta_{1}\right) \subseteq \operatorname{Sym}\left(R_{2}\right)$ and $\operatorname{Sym}\left(\zeta_{2}\right) \subseteq$ $\operatorname{Sym}\left(R_{1}\right), \operatorname{Sym}(R)=\operatorname{Sym}\left(R_{1}\right) \cup \operatorname{Sym}\left(R_{2}\right)$. Next, let $\kappa_{i}=\left(\kappa^{\prime} ; \zeta_{i}\right)\left\lceil\mathrm{FV}\left(P_{i}\right)\right.$, for $i \in\{1,2\}$. Since $P$ is linear, $\kappa_{1}$ and $\kappa_{2}$ have disjoint domains. Let $\kappa=\kappa_{1} \cup \kappa_{2}$. We define $Q=\left(Q_{1} Q_{2}\right)\{x \backslash R\}$. Now:

$$
Q=\left(Q_{1} Q_{2}\right)\{x \backslash R\} \rightarrow_{\mathrm{x}}\left(Q_{1}\{x \backslash R\}\right)\left(Q_{2}\{x \backslash R\}\right)=P_{1}\left[\kappa^{\prime} ; \zeta_{1}\right] P_{2}\left[\kappa^{\prime} ; \zeta_{2}\right]=P[\kappa]
$$

Also, suppose $X \in \operatorname{Dom}(\kappa)$. There are two subcases. If $X \in \operatorname{MV}\left(P_{2}\right)$, then $(\kappa ; \eta)(X)=\left(\kappa^{\prime} ; \zeta_{2} ; \eta\right)(X)=\left(\kappa^{\prime} ; \zeta^{\prime}\right)(X)=\zeta(X)$. Otherwise, $\kappa^{\prime}(X)=X$ and $\zeta^{\prime}(X)=\zeta(X)$. So $\kappa ; \eta=\zeta$. 
Finally, $\operatorname{Sym}(P)=\operatorname{Sym}\left(Q_{1}\right) \cup \operatorname{Sym}\left(Q_{2}\right) \cup \operatorname{Sym}\left(R_{1}\right) \cup \operatorname{Sym}\left(R_{2}\right)=\operatorname{Sym}\left(Q_{1}\right) \cup$ $\operatorname{Sym}\left(Q_{2}\right) \cup \operatorname{Sym}(R)=\operatorname{Sym}(Q)$, yielding the result of the (trm) case.

(ii) By induction on the length of the reduction sequence $M \rightarrow_{\mathrm{Bx}} P[\zeta]$. In the base case, if $M=P[\zeta]$, we take $Q=P$ and $\eta=\zeta$, satisfying the lemma with $\kappa=$ $\emptyset$. Otherwise, let $M \rightarrow_{\mathrm{Bx}} N \rightarrow_{\mathrm{Bx}} P[\zeta]$. We apply item (i), obtaining a passive $\lambda \mathrm{x}$-term $P^{\prime}$ and grafting $\zeta^{\prime}$ such that $M=P^{\prime}\left[\zeta^{\prime}\right], \operatorname{Sym}\left(P^{\prime}\right)=\operatorname{Sym}(P)$ and either the (trm) or (sub) case applies. Then we apply the induction hypothesis on the reduction $M \rightarrow_{\mathrm{Bx}} P^{\prime}\left[\zeta^{\prime}\right]$, obtaining a passive $\lambda \mathrm{x}$-term $Q$ and grafting $\eta$ such that $M=Q[\eta], \operatorname{Sym}(Q)=\operatorname{Sym}\left(P^{\prime}\right)(=\operatorname{Sym}(P)), Q \rightarrow_{\mathrm{Bx}} P^{\prime}[\kappa]$ for some grafting $\kappa$ such that $\kappa ; \eta \rightarrow{ }_{\mathrm{Bx}} \zeta^{\prime}$. Now we distinguish the following cases:

- (trm) If $P^{\prime} \rightarrow_{\mathrm{Bx}} P\left[\kappa_{0}\right]$, for some $\kappa_{0}$ such that $\kappa_{0} ; \zeta^{\prime}=\zeta$, then $Q \rightarrow_{\mathrm{Bx}}$ $P^{\prime}[\kappa] \rightarrow_{\mathrm{Bx}} P\left[\kappa_{0} ; \kappa\right]$ and $\kappa_{0} ; \kappa ; \eta \rightarrow_{\mathrm{Bx}} \kappa_{0} ; \zeta^{\prime}=\zeta$, as required.

- (sub) If $P^{\prime}=P$ and $\zeta^{\prime} \rightarrow_{\mathrm{x}} \zeta$, then $Q \rightarrow_{\mathrm{Bx}} P^{\prime}[\kappa]=P[\kappa]$ and $\kappa ; \eta \rightarrow_{\mathrm{Bx}} \zeta^{\prime} \rightarrow_{\mathrm{x}}$ $\zeta$, as required.

Example 4.7. Consider the Bx-reduction:

$$
(\lambda x \cdot \mathrm{g}(x, x))(\mathrm{f}(\mathrm{a})) \rightarrow_{\mathrm{Bx}} \mathrm{g}(\mathrm{f}(\mathrm{a}), \mathrm{f}(\mathrm{a}))
$$

and the prefix $P=\mathrm{g}(\mathrm{f}(X), Y)$ of the target. The suffix is $\zeta=[X \mapsto \mathrm{a}, Y \mapsto \mathrm{f}(\mathrm{a})]$. We can take:

$$
\begin{aligned}
& -Q=(\lambda x \cdot \mathrm{g}(x, x))(\mathrm{f}(Y)), \\
& -\kappa=[Y \mapsto \mathrm{f}(X)] \text { and } \\
& -\eta=[X \mapsto \mathrm{a}],
\end{aligned}
$$

satisfying the conditions of Theorem 4.6 (ii).

\subsection{Translating between Terms, Preterms and $\lambda \mathrm{x}$-Terms}

We are now dealing with three types of terms: terms, preterms and $\lambda \mathrm{x}$-terms. In this subsection we develop the following translating operations between them:

.$- b$ and $\sharp$ from terms to preterms and back;

- $\ominus^{\ominus}$ and ${ }^{\oplus}$ from preterms to $\lambda \mathrm{x}$-terms and back.

Translating between terms and preterms. We introduce a pair of operations which interpret terms as preterms, and vice versa: with $s^{b}$ (s-flat), we denote (the unique representative of) the term $s$, interpreted as a preterm, and with $s^{\sharp}$ ( $M$-sharp), we denote (the $\beta \bar{\eta}$-normal form of) the preterm $s$, interpreted as a higher-order term. These operations naturally generalize to substitutions. We will call a preterm $s$ a (fully extended//linear/local) $\bar{x}$-prepattern, if it is in $\beta \bar{\eta}$-normal form, and $s^{\sharp}$ is a (linear/fully extended/local) $\bar{x}$-pattern. 
Translating between preterms and $\lambda \mathrm{x}$-terms. We introduce the operations $\cdot \frac{\ominus}{x}$ and $\bullet^{\oplus}$, which map $\lambda$-terms to $\lambda \mathrm{x}$-terms ${ }^{1}$, and vice versa, as follows:

$$
\begin{aligned}
y_{\bar{x}} & =Y \text { if } y \notin \bar{x} & (Y \bar{\sigma})_{\mathrm{N}}^{\oplus} & =y \\
x & =x \text { if } x \in \bar{x} & x_{\mathrm{N}}^{\oplus} & =x \\
f_{\bar{x}}^{\ominus} & =f & f_{\mathrm{N}}^{\oplus} & =f \\
(\lambda y \cdot s)_{\bar{x}}^{\ominus} & =\lambda y \cdot s_{\bar{x} y}^{\ominus} & \left(\lambda y \cdot M_{\mathrm{N}}\right. & =\lambda y \cdot M^{\oplus} \\
\left(s_{1} s_{2}\right) \frac{\Theta}{x} & =\left(s_{1}\right) \frac{\Theta}{x}\left(s_{2}\right) \frac{\ominus}{\bar{x}} & & \left(M_{1} M_{2}\right)_{\mathrm{N}}^{\oplus}=M_{1}^{\oplus} M_{2}^{\oplus}
\end{aligned}
$$

Note that $\oplus$ also normalizes the term to x-normal form and removes explicit substitutions, and that, for each preterm $s$ and sequence of variables $\bar{x},\left(s \frac{\ominus}{x}\right)^{\oplus}=s$. The operations above are naturally generalized to translations between substitutions and graftings.

Lemma 4.8. Let $M, N$ be $\lambda \mathrm{x}$-terms. $M \rightarrow_{\mathrm{Bx}} N$ if and only if $M^{\oplus} \rightarrow_{\beta} N^{\oplus}$.

Proof. $(\Rightarrow)$ and $(\Leftarrow)$ are proved by induction on the length of the reductions $M \rightarrow_{\mathrm{Bx}} N$ and $M^{\oplus} \rightarrow_{\beta} N^{\oplus}$, respectively.

Although the above lemma suggests that Bx-reduction in the $\lambda \mathrm{x}$-calculus can easily simulate $\beta$-reduction, there is still a problem: ${ }^{\oplus}$ does not distribute properly over grafting application. The problem is similar to the problem given on page 8 . Consider the $\lambda$ x-term $M:=(\lambda x \cdot f(Y))$ a and grafting $\zeta:=[Y \mapsto x]$. Now $M[\zeta]^{\oplus}=$ $(\lambda x . \mathrm{f}(x)) \mathrm{a}, M^{\oplus}=(\lambda x . \mathrm{f}(y))$ a. $\zeta^{\oplus}=[y \mapsto x]$. Note that $\left(M^{\oplus}\right)^{\left(\zeta^{\oplus}\right)}=\lambda z . \mathrm{f}(x)$, because substitutions are capture-avoiding, and thus $M[\zeta]^{\oplus} \neq_{\beta}\left(M^{\oplus}\right)^{\left(\zeta^{\oplus}\right)}$.

The solution is to add as arguments to the free variables of the preterms as many (bound) variables as necessary (or more) to make the distribution work. In the example above we would have $s=(\lambda x . \mathrm{f}(y(x)))$ a and $\sigma=[y \mapsto \lambda x . x]$. Now, $s$ and $\sigma$ are, in a way that will be formalized in the next definition, similar to $M$ and $\zeta$, but now $M[\zeta]^{\oplus}={ }_{\beta} s^{\sigma}$.

Definition 4.9. Let $M$ be a $\lambda \mathrm{x}$-term and $\zeta$ a grafting. A tuple $\langle s, \sigma\rangle$ of preterm and substitution is a $\lambda$-extension of $\langle M, \zeta\rangle$ if there are graftings $\theta_{1}, \theta_{2}$ such that:

- $s=M\left[\theta_{1}\right]^{\oplus}$ and $\sigma=\left(\theta_{2} ; \zeta\right)^{\oplus}$;

- for each $X \in \operatorname{MV}(M), \theta_{1}(X)=X(\bar{z})$ and $\theta_{2}(X)=\lambda \bar{z} \cdot X$, where $\bar{z}$ is a list of variables containing at least the bound variables of $M$ in scope that occur in $\zeta(X)$ (in arbitrary order).

The notion of $\lambda$-extension is, again, naturally generalized to graftings and substitutions as the first component of the tuples.

\footnotetext{
1 Actually, the operation $\frac{\ominus}{x}$ is not a mapping, because $\alpha$-equivalent preterms preterms are identified as usual, and thus identical preterms can be mapped to different $\lambda \mathrm{x}$ terms. This is not a problem in practice, and can be fixed in theory by considering only preterms in " $\alpha$-normal form", e.g. by consecutively numbering the bound variables from left to right.
} 
Lemma 4.10. Let $\langle s, \sigma\rangle$ be a $\lambda$-extension of $\langle M, \zeta\rangle$. Then:

(i) $s^{\sigma}={ }_{\beta} M[\zeta]^{\oplus}$;

(ii) for each $\lambda \mathrm{x}$-term $N$ such that $M \rightarrow{ }_{\mathrm{Bx}} N, s^{\sigma}={ }_{\beta} N[\zeta]^{\oplus}$.

Proof. (i) Follows from the fact that for all $X \in \mathrm{MV}(M)$ it holds that

$$
\left(\theta_{1} ; \theta_{2}\right) \downarrow_{\mathrm{X}}(X)=X\left\{z_{1} \backslash z_{1}\right\} \cdots\left\{z_{n} \backslash z_{n}\right\}
$$

and thus $s^{\sigma}={ }_{\beta} M\left[\theta_{1} ; \theta_{2} ; \zeta\right]^{\oplus}={ }_{\beta} M[\zeta]^{\oplus}$.

(ii) Assume that $M \rightarrow \mathrm{Bx} N$. We take $t=N\left[\theta_{1}\right]^{\oplus}$ and show: (a) $s \rightarrow_{\beta} t$; and (b) $t^{\sigma}={ }_{\beta} N[\zeta]^{\oplus}$. Claim (a) follows from Lemma 4.8 and the fact that $M\left[\theta_{1}\right] \rightarrow \mathrm{Bx} N\left[\theta_{1}\right]$, because $M \rightarrow \mathrm{Bx} N$ by assumption. For (b) we must observe that, by definition, if $N_{1}\left\{x \backslash N_{2}\right\}$ is a subterm of $N$, then for all $X \in \operatorname{MV}\left(N_{1}\right), x$ does only occur bound in $\theta_{2} ; \zeta$, and thus $\left\ulcorner M\left[\theta_{1}\right] \downarrow_{\mathrm{x}}\left[\left(\theta_{2} ; \zeta\right) \downarrow_{\mathrm{x}}\right]\right\urcorner=\left\ulcorner M\left[\theta_{1} ; \theta_{2} ; \zeta\right] \downarrow_{\mathrm{x}}\right\urcorner$, where $\ulcorner K\urcorner$ denotes $K$ with all explicit substitutions removed, for arbitrary $K$. Therefore $t^{\sigma}={ }_{\beta} N\left[\theta_{1} ; \theta_{2} ; \zeta\right]^{\oplus}={ }_{\beta} N[\zeta]^{\oplus}$, and since $s \rightarrow_{\beta} t$ by $(\mathrm{a}), s^{\sigma}={ }_{\beta} N[\zeta]^{\oplus}$, as required.

The lemma works, because the arguments of the free variables in the term and the leading abstractions in the substitution, take over the role of the explicit substitutions, as can be seen in the following example:

Example 4.11. Let $M=(\lambda x .(\lambda y . Z) \mathrm{b})$ a be a $\lambda$ x-term, and $\zeta=[Z \mapsto \mathrm{f}(x, y)]$ a grafting. Now, according to Def. $4.9,\langle s, \sigma\rangle$, where $s=(\lambda x .(\lambda y . z(x, y)) \mathrm{b}) \mathrm{a}$ and $\sigma=[z \mapsto \lambda x y \cdot \mathrm{f}(x, y)]$ is a $\lambda$-extension of $\langle M, \zeta\rangle$, with, $\theta_{1}=[Z \mapsto Z(x, y)]$ and $\theta_{2}=[\lambda x y . Z]$. We check both cases of Lemma 4.10:

(i) $s^{\sigma}=(\lambda x \cdot(\lambda y \cdot(\lambda x y \cdot \mathrm{f}(x, y))(x, y)) \mathrm{b}) \mathrm{a}={ }_{\beta}(\lambda x \cdot(\lambda y \cdot \mathrm{f}(x, y)) \mathrm{b}) \mathrm{a}=M[\zeta]^{\oplus}$.

(ii) Let $N=Z\{y \backslash \mathrm{b}\}\{x \backslash \mathrm{a}\}$. Then $M \rightarrow_{\mathrm{x}} N$. Let $t=z(\mathrm{a}, \mathrm{b})$. Now $t^{\sigma}=\beta$ $\mathrm{f}(\mathrm{a}, \mathrm{b})=M[\zeta]^{\oplus}$. Since $s={ }_{\beta} t$, this means that $s^{\sigma}={ }_{\beta} M[\zeta]^{\oplus}$, as required. (Note that the $\oplus$ operation also reduces to $\mathrm{x}$-normal form.)

Translating patterns. Now we define a translation between pairs of prepatterns and presubstitutions, on the one hand, and linear, passive $\lambda \mathrm{x}$-terms and graftings on the other. Because the notion of pattern is parametrized by a sequence of variables, the translation operations are so as well.

Definition 4.12. Let $\mathbf{P}_{\bar{x}}^{+}$, a mapping which maps pairs of linear, passive $\lambda \mathrm{x}$-terms containing no explicit substitutions and graftings to pairs of local $\bar{x}$ prepatterns and substitutions, be defined as follows:

$$
\begin{array}{rlrl}
\mathbf{P}_{\bar{x}}^{+}\langle Y,[Y \mapsto M]\rangle & =\left\langle y(\bar{x}),\left[y \mapsto \lambda \bar{x} \cdot M^{\oplus}\right]\right\rangle \\
\mathbf{P}_{\bar{x}}^{+}\left\langle y\left(\overline{P_{n}}\right), \zeta\right\rangle & =\left\langle y\left(\overline{p_{n}}\right), \sigma\right\rangle & & \text { if } \mathbf{P}_{\bar{x}}^{+}\left\langle P_{i}, \zeta\left\lceil P_{i}\right\rangle=\left\langle p_{i}, \sigma\left\lceil p_{i}\right\rangle\right.\right. \\
\mathbf{P}_{\bar{x}}^{+}\left\langle f\left(\overline{P_{n}}\right), \zeta\right\rangle & =\left\langle f\left(\overline{p_{n}}\right), \sigma\right\rangle & & \text { if } \mathbf{P}_{\bar{x}}^{+}\left\langle P_{i}, \zeta\left\lceil P_{i}\right\rangle=\left\langle p_{i}, \sigma\left\lceil p_{i}\right\rangle\right.\right. \\
\mathbf{P}_{\bar{x}}^{+}\langle\lambda y \cdot P, \zeta\rangle & =\langle\lambda y \cdot p, \sigma\rangle & & \text { if } \mathbf{P}_{\bar{x} y}^{+}\langle P, \zeta\rangle=\left\langle p, \zeta_{\star}\right\rangle
\end{array}
$$

where $\zeta\lceil P$ denotes the restriction of grafting/substitution $\zeta$ to the metavariables/variables of $\lambda \mathrm{x}$-term $/$ preterm $P$. With $\mathbf{P}_{\bar{x}}^{-}$we denote the inverse of $\mathbf{P}_{\bar{x}}^{+} .^{2}$

\footnotetext{
${ }^{2}$ Like $\frac{\ominus}{x}$, the $\mathbf{P}_{\bar{x}}^{-}$operation is not actually a mapping, but this is no problem for the same reason. See footnote 1 .
} 
As usual, we generalize the above operations to (local pattern) substitutions and (linear, passive) graftings, in the obvious way. Note that the first element of the result of $\mathbf{P}_{\bar{x}}^{-}\langle\cdot, \cdot\rangle$ and $\mathbf{P}_{\bar{x}}^{+}\langle\cdot, \cdot\rangle$ does not depend on the second.

Lemma 4.13. Let $P$ be a linear, passive $\lambda \mathrm{x}$-term and $\zeta$ a grafting, both containing no explicit substitutions. Then $\mathbf{P}_{\bar{x}}^{+}\langle P, \zeta\rangle$ is a $\lambda$-extension of $\langle P, \zeta\rangle$, for arbitrary list of variables $\bar{x}$.

Proof. By the fact that all bound variables in scope are added as arguments to the free variables.

Example 4.14. Consider the linear, local $\lambda \mathrm{x}$-terms $P=\mathrm{f}(\lambda x y \cdot \mathrm{g}(Z, x))$ and $Q=$ $\operatorname{map}(\lambda x . Z$, nil $)$, and the grafting $\zeta=[Z \mapsto \mathrm{f}(x)]$. Then:

$$
\begin{aligned}
& \mathbf{P}_{\emptyset}^{+}\langle P, \zeta\rangle=\langle\mathrm{f}(\lambda x y \cdot \mathrm{g}(z(x, y), x)),[z \mapsto \lambda x y \cdot \mathrm{f}(x)]\rangle \\
& \mathbf{P}_{\emptyset}^{+}\langle Q, \zeta\rangle=\langle\operatorname{map}(\lambda x . Z(x), \operatorname{nil}),[z \mapsto \lambda x \cdot \mathrm{f}(x)]\rangle
\end{aligned}
$$

\subsection{Proof of the Prefix Property}

We repeat the text of Theorem 4.1, for convenience:

Theorem 4.1 (Prefix Property). Let $\mathcal{H}^{\omega}$ be the $\omega$-labelling of a non-collapsing HRS, $s$ a term, $p$ a local $\bar{x}$-pattern and $\sigma$ a substitution. If $s \rightarrow \mathcal{H}^{\omega} p^{\sigma}$, then there exist a local $\bar{x}$-pattern $q$ and a substitution $\tau$, such that $s=q^{\tau}$, $\operatorname{fam}(p) \geq \operatorname{fam}(q)$, and either:

$-q \rightarrow \mathcal{H}^{\omega} p^{v}$, for some substitution $v$ such that $v ; \tau=\sigma$; or

(trm)

$-q=p$ and $\tau \rightarrow \mathcal{H}^{\omega} \sigma$.

Proof. In the course of this proof we use letters subscripted with a $\star$ (e.g. $\left.s_{\star}, P_{\star}, \sigma_{\star} \ldots\right)$ to denote preterms and presubstitutions. Letters without $\star$-subscript denote terms and substitutions on the term level, or $\lambda$ x-terms and graftings.

We prove the theorem by induction on the context of the step $s \rightarrow_{\mathcal{H}^{\omega}} p^{\sigma}$. If $p$ has a variable as head, then the (sub) case is trivially satisfied. If the step does not occur at the head, then the result follows easily from the induction hypothesis.

So, assume $s=l^{\rho}$ and $p^{\sigma}=r^{\rho}$, for some rule $\lambda \bar{x} . l \rightarrow \lambda \bar{x} . r \in R$ and substitution $\rho$. We cast everything down to the preterm level, i.e.: $s_{\star}:=s^{b}, l_{\star}:=l^{b}$, $\rho_{\star}:=\rho^{b}, r_{\star}:=r^{b}, p_{\star}:=p^{b}$ and $\sigma_{\star}:=\sigma^{b}$. Furthermore, let $\langle P, \zeta\rangle:=\mathbf{P}_{\bar{x}}^{-}\left\langle p_{\star}, \sigma_{\star}\right\rangle$. Now, by definition, $P$ is a linear, passive $\lambda$ x-term, and $P[\zeta]^{\oplus}=\beta p_{\star}^{\sigma_{\star}} . P[\zeta]$ is a Bx-normal form and $r_{\star}^{\rho_{\star}}={ }_{\beta} p_{\star}^{\sigma_{\star}}={ }_{\beta} P[\zeta]^{\oplus}$, so $r_{\star}^{\rho_{\star}} \rightarrow \beta P[\zeta]^{\oplus}$, and from this and Lemma 4.8 it follows that:

$$
R[\mu] \rightarrow_{\mathrm{Bx}} P[\zeta]
$$

where $R:=\left(r_{\star}\right) \frac{\ominus}{x}$ and $\mu:=\left(\rho_{\star}\right) \frac{\ominus}{x}$. Now, we can apply the $\lambda x$-Prefix Property (Theorem 4.6, ii) and obtain a linear, passive $\lambda$ x-term $P^{\prime}$, with $\operatorname{Sym}\left(P^{\prime}\right)=$ $\operatorname{Sym}(P)$, and graftings $\eta, \kappa$ such that:

$$
P^{\prime}[\eta]=R[\mu], \quad P^{\prime} \rightarrow \mathrm{Bx} P[\kappa] \quad \text { and } \quad \kappa ; \eta \rightarrow \mathrm{Bx} \zeta
$$


Because of the first equality above, we can apply Lemma 4.4 and obtain a linear, passive grafting $\mu^{\prime}$ and graftings $\eta^{\prime}, \kappa$ with $\operatorname{Sym}\left(\eta^{\prime}\right) \subseteq \operatorname{Sym}(R)$ and $\operatorname{Sym}\left(\mu^{\prime}\right) \subseteq$ $\operatorname{Sym}\left(P^{\prime}\right)$, such that:

$$
P^{\prime}\left[\eta^{\prime}\right]=R\left[\mu^{\prime}\right], \quad \eta^{\prime} ; \kappa=\eta \quad \text { and } \quad \mu^{\prime} ; \kappa=\mu
$$

Now the only thing we have to do, is translate the obtained $\lambda \mathrm{x}$-terms and graftings to terms and substitutions, using the techniques developped in the previous subsection. This is not hard, in principle, but a cumbersome operation.

Let $\left\langle\rho_{\star}^{\prime}, \tau_{\star}\right\rangle:=\mathbf{P}_{\emptyset}^{+}\left\langle\mu^{\prime}, \kappa\right\rangle$. Then $\rho_{\star}^{\prime}$ is a local pattern substition, and by Lemma 4.13, $\left\langle\rho_{\star}^{\prime}, \tau_{\star}\right\rangle$ is a $\lambda$-extension of $\left\langle\mu^{\prime}, \kappa\right\rangle$. Because the object variables of $r_{\star}$ and $\rho_{\star}^{\prime}$ are disjoint, it follows that $\left\langle r_{\star}^{\rho_{\star}^{\prime}}, \tau_{\star}\right\rangle$ is a $\lambda$-extension of $\left\langle R\left[\mu^{\prime}\right], \kappa\right\rangle$ and thus of $\left\langle P^{\prime}\left[\eta^{\prime}\right], \kappa\right\rangle$. Let $\theta$ be the grafting such that $\rho_{\star}^{\prime}=\left(\mu^{\prime} ; \theta\right)^{\oplus}$ and let $\eta^{\prime \prime}:=\eta^{\prime} ; \theta$. Next, let $\left\langle P_{\star}^{\prime}, \eta_{\star}^{\prime \prime}\right\rangle:=\mathbf{P}_{\bar{x}}^{+}\left\langle P^{\prime}, \eta^{\prime \prime}\right\rangle$. By definition, there exists a grafting $\theta^{\prime}$ such that $P_{\star}^{\prime}=P^{\prime}\left[\theta^{\prime}\right]^{\oplus}$, and because $P^{\prime} \rightarrow_{\mathrm{Bx}} P[\kappa]$ it is the case that $P_{\star}^{\prime}=P\left[\kappa ; \theta^{\prime}\right]^{\oplus}$. Now, let $\left\langle P_{\star}, \kappa_{\star}\right\rangle:=\mathbf{P}_{\bar{x}}^{+}\left\langle P, \kappa ; \theta^{\prime}\right\rangle$. Since $P_{\star}$ does not depend on $\kappa ; \theta^{\prime}, P_{\star}=p_{\star}$. We know now that $P_{\star}^{\kappa_{\star}}={ }_{\beta} P\left[\kappa ; \theta^{\prime}\right]^{\oplus}={ }_{\beta} P^{\prime}\left[\theta^{\prime}\right]^{\oplus}={ }_{\beta} P_{\star}^{\prime}$.

Finally, we take: $q_{\star}=l_{\star}^{\rho_{\star}^{\prime}}, v_{\star}=\kappa_{\star} ; \eta_{\star}^{\prime \prime}$. Then:

- $q_{\star}$ is a local $\bar{x}$-prepattern, because $l_{\star}$ is a local $\bar{x}$-prepattern and $\rho_{\star}^{\prime}$ is a local pattern substitution;

$-r_{\star}^{\rho_{\star}^{\prime}}={ }_{\beta} P_{\star}^{\prime \eta_{\star}^{\prime \prime}}=\beta P_{\star}^{\kappa_{\star} ; \eta_{\star}^{\prime \prime}}=\beta p_{\star}^{v_{\star}}$

$-s_{\star}=l_{\star}^{\rho_{\star}}={ }_{\beta} l_{\star}^{\rho_{\star}^{\prime} ; \tau_{\star}}={ }_{\beta} q_{\star}^{\tau_{\star}} ;$

$-v_{\star} ; \tau_{\star}=\beta \kappa_{\star} ; \eta_{\star}^{\prime \prime} ; \tau_{\star}={ }_{\beta} \sigma_{\star} ;$

$-\operatorname{fam}\left(p_{\star}\right)=\operatorname{fam}\left(P^{\prime}\right)=\operatorname{fam}\left(P^{\prime}\left[\eta^{\prime}\right]\right)=\operatorname{fam}\left(R\left[\rho^{\prime}\right]\right) \geq \operatorname{fam}\left(l_{\circ}\left[\rho^{\prime}\right]\right)=\operatorname{fam}\left(q_{\star}\right)$, where the second equality holds because $\operatorname{Sym}\left(\eta^{\prime}\right) \subseteq \operatorname{Sym}(r)$, all labels in $r$ are the same, $p$ and $r$ have at least one symbol in common because $r$ is non-collapsing, and thus $\operatorname{Sym}\left(\eta^{\prime}\right) \subseteq \operatorname{Sym}(p)$.

Now we take $q:=q_{\star}^{\sharp}, v:=v_{\star}^{\sharp}$ and $\tau:=\tau_{\star}$ to satisfy the (trm) case.

\section{$5 \quad$ Finite Family Developments}

In this section we apply the prefix property of the previous section to prove that all family developments of HRSs are finite. We restrict our attention to noncollapsing HRSs first. In the next section, we will describe a way to generalize the result to collapsing HRSs as well.

Families are formalized by labelling all function symbols with natural numbers, as defined in Def. 3.1. We prove that the resulting system is terminating if we restrict the labels to some finite bound. The proof is inspired by the proof by Van Oostrom [17]. The differences between this proof and the one by Van Oostrom are the following:

- We use a different method of labelling. Our labelling has the property that one step of the labelled HRS corresponds exactly to one step in the original. Also, our notion of labelling is an instance of the abstract notion of labelling put forth in $[18,14]$. 
- In Van Oostrom's paper, the proof of Lemma 15 is omitted. Here, we give a proof of that lemma (adapted for the different method of labelling) by reducing it to the Prefix Property.

Lemma 5.1. Let $\mathcal{H}^{\omega}$ be the labelling of a non-collapsing HRS, $s$ be a term, $p$ a local pattern, $\ell \in \mathbb{N}$ a label and $\tau$ and $\sigma$ substitutions such that for any $x \in \operatorname{Dom}(\sigma), \sigma(x)$ has a function symbol labelled with $\ell$ as head. If $s^{\sigma} \rightarrow \mathcal{H}^{\omega} p^{\tau}$, then either:

$$
\begin{aligned}
& -\operatorname{fam}(p) \geq \ell ; \text { or } \\
& -s \rightarrow \mathcal{H} p^{v}, \text { for some } v \text { such that } v ; \sigma \rightarrow \mathcal{H}^{\omega} \tau .
\end{aligned}
$$

Proof. By induction on the length of the reduction $s^{\sigma} \rightarrow \mathcal{H}^{\omega} p^{\tau}$. If the length is 0 , there are two cases: if a subtitution $v$ exists such that $s=p^{v}$, then the conditions of the (ext) case are trivially satisfied; otherwise, we show (int) by induction on $p$, using the assumption on the structure of $\sigma$ in the base case.

Otherwise, suppose $s^{\sigma} \rightarrow \mathcal{H}^{\omega} s^{\prime} \rightarrow \mathcal{H}^{\omega} p^{\tau}$. By Theorem 4.1, there exist a local pattern $q$ and substitution $\sigma^{\prime}$ such that $s^{\prime}=q^{\sigma^{\prime}}$, fam $(p) \geq \operatorname{fam}(q)$ and either (trm) $q \rightarrow \mathcal{H}^{\omega} p^{v^{\prime}}$ and $v^{\prime} ; \sigma^{\prime}=\tau$; or (sub) $p=q$ and $\sigma^{\prime} \rightarrow \mathcal{H}^{\omega} \tau$. Applying the induction hypothesis to $s^{\sigma} \rightarrow \mathcal{H}^{\omega} q^{\sigma^{\prime}}$ yields that one of the following cases must apply:

- (int) $\operatorname{fam}(q) \geq \ell$, but then fam $(p) \geq \ell$ by transitivity of $\geq$.

- (ext) $s \rightarrow \mathcal{H}^{\omega} q^{v}$ and $v ; \sigma \rightarrow \mathcal{H}^{\omega} \sigma^{\prime}$, for some substitution $v$. We distinguish the following cases:

- (trm) $s \rightarrow \mathcal{H}^{\omega} q^{v} \rightarrow \mathcal{H}^{\omega} p^{v^{\prime} ; v}$ and $v^{\prime} ; v ; \sigma \rightarrow \mathcal{H}^{\omega} v^{\prime} ; \sigma^{\prime}=\tau$.

- (sub) $s \rightarrow_{\mathcal{H}^{\omega}} q^{v}=p^{v}$ and $v ; \sigma \rightarrow \mathcal{H}^{\omega} \sigma^{\prime} \rightarrow_{\mathcal{H}^{\omega}} \tau$.

In both cases the (ext) case of the lemma is satisfied.

Theorem 5.2. Let $\mathcal{H}^{\omega}$ be the labelling of a non-collapsing HRS, and let $\mathcal{R}$ : $s_{1} \rightarrow \mathcal{H}^{\omega} s_{2} \rightarrow \mathcal{H}^{\omega} \cdots$ be a $\mathcal{H}^{\omega}$-reduction. $\mathcal{R}$ is finite, if and only if there is a $\ell_{\max } \in \mathbb{N}$ such that $\operatorname{fam}\left(s_{i}\right) \leq \ell_{\max }$ for all $s_{i}$.

Proof. $(\Rightarrow)$ : Trivial, because a finite reduction has a finite amount of labels.

$(\Leftarrow)$ : We prove the theorem by showing that $\mathcal{H}^{\omega}=\left\langle\Sigma^{\omega}, R^{\omega}\right\rangle$ is terminating if we restrict it to rules $l \rightarrow r \in R^{\omega}$ where fam $(r) \leq \ell_{\max }$. By the fact that an HRS is terminating if and only if $r^{\sigma}$ is terminating for every right-hand side $r$ and terminating substitution $\sigma$ [17, Lemma 8], it suffices to show termination of $\left(s^{\ell}\right)^{\sigma}$, for every non-(subst-subst)-collapsing ${ }^{3}$, term $s$, label $\ell \in \mathbb{N}$ and terminating substitution $\sigma$. We do this by induction on $\left(\ell_{\max }-\ell\right)$.

Let $\left(s^{\ell}\right)^{\sigma}$ be a minimal non-terminating term. Since it is minimal, we can assume that this infinite reduction contains at least one head step, and $s=$ $a\left(s_{1}, \ldots, s_{n}\right)$, so $s^{\ell}=a^{\prime}\left(\left(s_{1}^{\ell}\right)^{\sigma}, \ldots,\left(s_{n}^{\ell}\right)^{\sigma}\right)$. By minimality, the $s_{i}^{\ell}$ are terminating. We distinguish the following cases:

\footnotetext{
${ }^{3}$ We drop the (context-subst) condition of Def 2.5, because subterms of non (contextsubst)-collapsing terms can be (constext-subst)-collapsing, meaning that an infinite reduction from a minimal counter example might not contain a head step.
} 
- If $a$ is the function symbol $f$, then $a^{\prime}=f^{\ell}$. Since the first head step strictly increases the label, termination follows from the induction hypothesis.

- If $a$ is a variable, then it must be in the domain of $\sigma$ (otherwise, a head step would not be possible, contradicting minimality). Suppose:

$$
\sigma(a)=\lambda \bar{x} \cdot b\left(t_{1}, \ldots, t_{m}\right)
$$

and thus $\left(s^{\ell}\right)^{\sigma}=b\left(t_{1}^{\sigma^{\prime}}, \ldots, t_{m}^{\sigma^{\prime}}\right)$, where $\sigma^{\prime}=\left[x_{1} \mapsto s_{1}^{\ell}, \ldots, x_{n} \mapsto s_{n}^{\ell}\right]$. We prove the lemma by a nested induction on the order $\ll_{\mathcal{H}^{\omega}}$ starting from $\sigma(a)$. We distinguish the following cases:

- Suppose $b=f^{\ell^{\prime}}$. Then an infinite reduction from $\left(s^{\ell}\right)^{\sigma}$ looks like:

$$
f^{\ell^{\prime}}\left(t_{1}^{\sigma^{\prime}}, \ldots, t_{m}^{\sigma^{\prime}}\right) \rightarrow \mathcal{H}^{\omega} f^{\ell^{\prime}}\left(t_{1}, \ldots, t_{m}\right)=l^{\tau} \rightarrow \mathcal{H}^{\omega}\left(r^{\ell^{\prime \prime}}\right)^{\tau} \rightarrow \mathcal{H}^{\omega} \cdots
$$

where $l \rightarrow r^{\ell^{\prime}} \in R^{\omega}$. Since $a$ is a variable and $s$ is not (subst-subst)collapsing, we know that the $s_{i}^{\ell}$ have function symbols labelled with $\ell$ as head, and we can apply Lemma 5.1 to $l^{\tau}$ :

* (int): $\operatorname{fam}(l) \geq \ell$ : It follows by the fact that, by construction, $\ell^{\prime \prime}=$ fam $(l)+1$, that $\ell^{\prime \prime} \geq \ell$. Thus, by the outermost induction hypothesis, $\left(r^{\ell^{\prime \prime}}\right)^{\tau}$ is terminating, contradicting the assumption that $s$ is not.

* (ext): $a^{\sigma} \rightarrow \mathcal{H}^{\omega} l^{v}$ and $v ; \sigma^{\prime} \rightarrow_{\mathcal{H}} \omega \tau$ : We know that:

$$
a^{\sigma} \rightarrow \mathcal{H}^{\omega} l^{v} \rightarrow \mathcal{H}^{\omega}\left(r^{\ell^{\prime \prime}}\right)^{v} \quad \text { and } \quad\left(r^{\ell^{\prime \prime}}\right)^{(v ; \sigma)} \rightarrow \mathcal{H}^{\omega}\left(r^{\ell^{\prime \prime}}\right)^{\tau}
$$

Since the left reduction consists of at least one step (the last one), $\left(r^{\ell^{\prime \prime}}\right)^{v}$ is terminating by the nested induction hypothesis. By the right sequence and the fact that $\sigma^{\prime}$ is terinating, this yields termination of $\left(r^{\ell^{\prime \prime}}\right)^{\tau}$, contradicting the assumption that $s$ is not..

- Suppose $b$ is a variable, it must be in the domain of $\sigma$. Suppose $b=x_{i}$, and $s_{i}=\lambda \bar{y} \cdot c\left(u_{1}, \ldots, u_{l}\right)$. Then $\left(s^{\ell}\right)^{\sigma}=c\left(u_{1}, \ldots, u_{l}\right)^{(\sigma ; \tau)}$ where $\tau=$ $\bigcup_{1 \leq i \leq m}\left[y_{i} \mapsto t_{i}^{\sigma^{\prime}}\right]$. Now $\sigma$ is terminating by assumption, and the $t_{i}^{\sigma^{\prime}}$ are terminating by the nested induction hypothesis, so $\sigma ; \tau$ is a terminating substitution. Since $c\left(u_{1}, \ldots, u_{l}\right)$ is a proper subterm of $s$, termination of $c\left(u_{1}, \ldots, u_{l}\right)^{(\sigma ; \tau)}$ follows by minimality.

\section{Dealing with Collapsing HRSs}

In the previous sections we restricted our attention to non-collapsing HRSs. Both the Prefix Property and FFD do not hold for collapsing HRSs, as is witnessed by the following two counterexamples:

Example 6.1 (Prefix Property). Consider the collapsing HRS Mu:

$$
\mathrm{mu}(\lambda x . z(x)) \rightarrow z(\mathrm{mu}(\lambda x . z(x))
$$

and the following $\mathcal{M} u^{\omega}$-step:

$$
\mathrm{mu}^{3}\left(\lambda x . \mathrm{f}^{2}(x)\right) \rightarrow \mathcal{M} u^{\omega} \mathrm{f}^{2}\left(\mathrm{mu}^{4}\left(\lambda x . \mathrm{f}^{2}(x)\right)\right)
$$

It is easy to check that the prefix $p=\mathrm{f}^{2}(u)$ of the target of the step has no ancestor $q$ that satisfies the requirements of the Prefix Property (Theorem 4.1). 
Example 6.2 (FFD). Consider the collapsing HRS L Lam:

$$
\operatorname{app}(\operatorname{lam}(\lambda x . z(x), y)) \rightarrow z(y)
$$

Then one $\mathcal{L} a m^{\omega}$-step is the following:

$$
\begin{aligned}
& \operatorname{app}^{1}\left(\operatorname{lam}^{1}\left(\lambda x \cdot \operatorname{app}^{1}(x, x)\right), \operatorname{lam}^{1}\left(\lambda x \cdot \operatorname{app}^{1}(x, x)\right)\right) \\
& \rightarrow \mathcal{L} a m^{\omega} \operatorname{app}^{1}\left(\operatorname{lam}^{1}\left(\lambda x \cdot \operatorname{app}^{1}(x, x)\right), \operatorname{lam}^{1}\left(\lambda x \cdot \operatorname{app}^{1}(x, x)\right)\right)
\end{aligned}
$$

So we see that $\mathcal{L} a m^{\omega}$ has a one-step cycle, and thus an infinite reduction with bounded labels.

The problem in both cases is that, because of applying a collapsing rule, a function symbol can be directly connected to a previously unconnected function symbol from the context or substitution, or to the root of the term, without the rule leaving any trace in between, in the form of a label. This can be remedied by including "empty" function symbols, named $\epsilon_{\alpha}$, for each base type $\alpha$, in the right-hand sides of rules, and "saturating" the left-hand sides of rules with those empty function symbols. The same approach is taken for the first-order case in [14, Chap. 8].

\section{Definition 6.3 ( $\epsilon$-lifting).}

(i) The $\epsilon$-lifting $\Sigma^{\epsilon}$ of a signature $\Sigma$, consists of all function symbols of $\Sigma$, and, for each base type $\alpha$, a function symbol $\epsilon_{\alpha}: \alpha \rightarrow \alpha$.

(ii) The $\epsilon$-lifting of a term $s$ of type $\alpha$, written $s^{\epsilon}$, is defined as follows:

$$
\begin{aligned}
x\left(s_{1}, \ldots, s_{n}\right)^{\epsilon} & \left.=\epsilon_{\alpha}\left(x\left(s_{1}^{\epsilon}, \ldots, s_{n}^{\epsilon}\right)\right)\right) \\
f\left(s_{1}, \ldots, s_{n}\right)^{\epsilon} & =f\left(s_{1}^{\epsilon}, \ldots, s_{n}^{\epsilon}\right) \\
\left(\lambda x . s_{0}\right)^{\epsilon} & =\lambda x . s_{0}^{\epsilon}
\end{aligned}
$$

(iii) The projection operator $|\cdot|_{\epsilon}$ maps $\Sigma^{\epsilon}$-terms to $\Sigma$-terms by removing all $\epsilon_{\alpha}$-symbols.

(iv) The set of $\epsilon$-saturations of a pattern $p$, denoted by $\operatorname{Sat}^{\epsilon}(s)$, is defined as $\operatorname{Sat}^{\epsilon}(p)=\operatorname{Sat}_{\text {out }}^{\epsilon}(p)$, where:

$$
\begin{aligned}
\operatorname{Sat}_{\text {out }}^{\epsilon}\left(x\left(\overline{y_{n}}\right)\right) & =\left\{x\left(\overline{y_{n}}\right)\right\} \\
\operatorname{Sat}_{\text {out }}^{\epsilon}\left(f\left(\overline{p_{n}}\right)\right) & =\left\{f\left(\overline{q_{n}}\right) \mid q_{i} \in \operatorname{Sat}_{\text {in }}^{\epsilon}\left(p_{i}\right)\right\} \\
\operatorname{Sat}_{\text {out }}^{\epsilon}\left(\lambda x \cdot p_{0}\right) & =\left\{\lambda x \cdot q_{0} \mid q_{0} \in \operatorname{Sat}_{\text {in }}^{\epsilon}\left(p_{0}\right)\right\} \\
\operatorname{Sat}_{\text {in }}^{\epsilon}\left(x\left(\overline{y_{n}}\right)\right) & =\left\{x\left(\overline{y_{n}}\right)\right\} \\
\operatorname{Sat}_{\text {in }}^{\epsilon}\left(f\left(\overline{p_{n}}\right)\right) & =\left\{\epsilon_{\alpha}^{m}\left(f\left(\overline{q_{n}}\right)\right) \mid m \in \mathbb{N}, q_{i} \in \operatorname{Sat}_{\text {in }}^{\epsilon}\left(p_{i}\right)\right\} \\
\operatorname{Sat}_{\text {in }}^{\epsilon}\left(\lambda x \cdot p_{0}\right) & =\left\{\lambda x \cdot q_{0} \mid q_{0} \in \operatorname{Sat}_{\text {in }}^{\epsilon}\left(p_{0}\right)\right\}
\end{aligned}
$$

where $\alpha$ is the type of $p$, and $\epsilon_{\alpha}^{m}(p)$ is inductively defined by $\epsilon_{\alpha}^{0}(p)=p$, $\epsilon_{\alpha}^{m+1}(p)=\epsilon_{\alpha}\left(\epsilon_{\alpha}^{m}(p)\right)$.

(v) The $\epsilon$-lifting of an HRS $\mathcal{H}=\langle\Sigma, R\rangle$ is defined as $\mathcal{H}^{\epsilon}=\left\langle\Sigma^{\epsilon}, R^{\epsilon}\right\rangle$, where

$$
R^{\epsilon}=\bigcup_{l \rightarrow r \in R}\left\{l^{\prime} \rightarrow r^{\epsilon} \mid l^{\prime} \in \operatorname{Sat}^{\epsilon}(l)\right\}
$$


The $\epsilon$-liftings of the two counter examples introduced earlier in this section do not have the same problems as their originals:

Example 6.4. The $\epsilon$-lifting of $\mathcal{M} u$ (types of $\epsilon$ 's omitted):

$$
\operatorname{mu}(\lambda x . z(x)) \rightarrow \epsilon(z(\epsilon(\operatorname{mu}(\lambda x . \epsilon(z(\epsilon(x)))))))
$$

A $\left(\mathcal{M} u^{\epsilon}\right)^{\omega}$ step corresponding to the step of Ex. 6.1 is:

$$
\mathrm{mu}^{3}\left(\lambda x . \mathrm{f}^{2}(x)\right) \rightarrow\left(\mathcal{M} u^{\epsilon}\right)^{\omega} \epsilon^{4}\left(\mathbf{f}^{2}\left(\epsilon^{4}\left(\mathrm{mu}^{4}\left(\lambda x . \epsilon\left(\mathbf{f}^{2}(\epsilon(x))\right)\right)\right)\right)\right)
$$

Take the corresponging prefix $p=\epsilon^{4}\left(\mathrm{f}^{2}(y)\right)$. Now, the Prefix Property is satisfied with $q=\mathrm{mu}^{3}\left(\lambda x . \mathrm{f}^{2}(x)\right), \tau=\emptyset$ and $v=\left[z \mapsto \epsilon^{4}\left(\mathrm{mu}^{4}\left(\lambda x . \epsilon\left(\mathrm{f}^{2}(\epsilon(x))\right)\right)\right)\right]$.

Example 6.5. The $\epsilon$-lifting of $\mathcal{L}$ am consists of (among others) the following rules:

$$
\begin{aligned}
\operatorname{app}(\operatorname{lam}(\lambda x . z(x), y)) & \rightarrow \epsilon(z(\epsilon(y))) \\
\operatorname{app}(\epsilon(\operatorname{lam}(\lambda x . z(x))), y) & \rightarrow \epsilon(z(\epsilon(y))) \\
\operatorname{app}(\epsilon(\epsilon(\operatorname{lam}(\lambda x . z(x)))), y) & \rightarrow \epsilon(z(\epsilon(y)))
\end{aligned}
$$

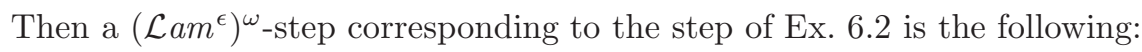

$$
\begin{aligned}
& \operatorname{app}^{1}\left(\operatorname{lam}^{1}\left(\lambda x \cdot \operatorname{app}^{1}(x, x)\right), \operatorname{lam}^{1}\left(\lambda x \cdot \operatorname{app}^{1}(x, x)\right)\right) \\
& \rightarrow\left(\mathcal{L a m}^{\epsilon}\right)^{\omega} \epsilon^{2}\left(\operatorname{app}^{1}\left(\epsilon^{2}\left(\operatorname{lam}^{1}\left(\lambda x \cdot \operatorname{app}^{1}(x, x)\right)\right), \epsilon^{2}\left(\operatorname{lam}^{1}\left(\lambda x \cdot \operatorname{app}^{1}(x, x)\right)\right)\right)\right)
\end{aligned}
$$

Now, all redex patterns have a maximum label of 2 , instead of 1 .

We can show that $\epsilon$-lifting does not interfer with rewriting:

Lemma 6.6. Let $\mathcal{H}$ be an HRS and $\mathcal{H}^{\epsilon}$ its $\epsilon$-lifting. Then:

(i) Let $s, t$ be $\mathcal{H}$-terms such that $s \rightarrow \mathcal{H}$ t. For each $\mathcal{H}^{\epsilon}$-term $s^{\prime}$ such that $\left|s^{\prime}\right|_{\epsilon}=$ $s$ there exists a unique $\mathcal{H}^{\epsilon}$-term $t^{\prime}$ such that $\left|t^{\prime}\right|_{\epsilon}=t$ and $s^{\prime} \rightarrow_{\mathcal{H}^{\epsilon}} t^{\prime}$.

(ii) Let $s, t$ be $\mathcal{H}^{\epsilon}$-terms. If $s \rightarrow \mathcal{H}^{\epsilon} t$, then $|s|_{\epsilon} \rightarrow_{\mathcal{H}}|t|_{\epsilon}$.

Proof. (i) is proved by induction on the step, noting that the type of the $\epsilon$ 's prohibits them from interferring with $\beta$-equivalence, and (ii) follows from the facts that, for each $s$, there is an $s^{\prime} \in \operatorname{Sat}^{\epsilon}(s)$ such that $s^{\epsilon}$ matches a subterm of $s^{\prime}$, and that, for any term $t$, if $t \in \operatorname{Sat}^{\epsilon}(s)$, then $|t|_{\epsilon}=s$.

Proposition 6.7. For any $H R S \mathcal{H}$, its $\epsilon$-lifting $\mathcal{H}^{\epsilon}$ is a non-collapsing HRS.

Proof. It is easy to see, that for all collapsing terms $s, s^{\epsilon}$ is non-collapsing.

Theorem 6.8 (FFD). Let $\left(\mathcal{H}^{\epsilon}\right)^{\omega}$ be the $\epsilon \omega$-labelling of an HRS, and let $\mathcal{R}$ : $s_{1} \rightarrow\left(\mathcal{H}^{\epsilon}\right)^{\omega} s_{2} \rightarrow\left(\mathcal{H}^{\epsilon}\right)^{\omega} \cdots$ be a $\left(\mathcal{H}^{\epsilon}\right)^{\omega}$-reduction. $\mathcal{R}$ is finite, if and only if there is $a \ell_{\max } \in \mathbb{N}$ such that $\operatorname{fam}\left(s_{i}\right) \leq \ell_{\max }$ for all $s_{i}$.

Proof. By Prop. 6.7 and Theorem 5.2. 


\section{Applications and Further Research}

The Prefix Property and Finite Family Developments are useful tools for proving various properties of HRSs. For example, an alternative proof of termination of the simply typed $\lambda$-calculus (encoded as an HRS) uses FFD. Also, in a work in progress by the author, FFD is used to prove the termination of a higher-order standardization procedure. This result can be used to formalize the notion of equivalence of reductions, in a similar way as is done in [14].

For future research, it might be interesting to further investigate the relation between FFD and the Dependency Pair method [1],one of the most powerful (first-order) termination techniques of today, both in the higher-order and firstorder case. Since FFD and the Dependency Pair method both essentially depend on the same principle, that an infinite reduction must have an unbounded creation depth, it the author's conjecture that FFD, or the Prefix Property, can be used to design a higher-order Dependency Pair method.

\section{A Termination of the Simply Typed $\lambda$-Calculus}

The most well-known proof of termination of the simply typed $\lambda$-calculus is the proof using strong computability due to Tait [13]. Here we present a termination proof of an HRS which encodes the simply typed $\lambda$-calculus, using the Finite Family Developments result of the present paper. ${ }^{4}$

The HRS we consider has an infinite number of rules. Let $\alpha, \beta$ range over (codes of) types, and let the following signature be given:

$$
\begin{aligned}
& \mathrm{T}_{\alpha}: \text { term } \rightarrow \text { term } \\
& \text { app }: \text { term } \rightarrow \text { term } \rightarrow \text { term } \\
& \text { lam }:(\text { term } \rightarrow \text { term }) \rightarrow \text { term }
\end{aligned}
$$

The simply typed $\lambda$-calculus can be encoded as the following HRS, $\mathcal{L} a m \rightarrow$ :

$$
\operatorname{app}\left(\mathrm{T}_{\alpha \rightarrow \beta}^{n}(\operatorname{lam}(\lambda x \cdot Z(x))), Y\right) \rightarrow \mathrm{T}_{\beta}\left(Z\left(\mathrm{~T}_{\alpha}(Y)\right)\right)
$$

for every $n>0$, where $f^{n}(s)$ is defined (here) as: $f^{0}(s)=s$ and $f^{n+1}(s)=$ $f\left(f^{n}(s)\right)$. Termination of $\mathcal{L} a m \rightarrow$ is proved in Prop. A.3. But first, we show that the HRS encodes the simply typed $\lambda$-calculus in the following sense: every reduction in the simply typed $\lambda$-calculus can be lifted to a $\mathcal{L} a m \rightarrow$-reduction. For this, we use the following map h, which maps each simply typed $\lambda$-term to a set of possible $\mathcal{L} a m \rightarrow$-encodings of it:

$$
\begin{aligned}
\mathrm{h}\left(x_{\alpha}\right) & =\left\{\mathrm{T}_{\alpha}^{n}(x) \mid n>0\right\} \\
\mathrm{h}\left(f_{\alpha}\right) & =\left\{\mathrm{T}_{\alpha}^{n}(f) \mid n>0\right\} \\
\mathrm{h}\left(\lambda x_{\alpha} \cdot M_{\beta}\right) & =\left\{\mathrm{T}_{\alpha \rightarrow \beta}^{n}(\operatorname{lam}(\lambda x . s)) \mid s \in \mathrm{h}(M), n>0\right\} \\
\mathrm{h}\left(M_{\alpha \rightarrow \beta} N_{\alpha}\right) & =\left\{\mathrm{T}_{\beta}^{n}(\operatorname{app}(s, t)) \mid s \in \mathrm{h}(M), t \in \mathrm{h}(N), n>0\right\}
\end{aligned}
$$

\footnotetext{
${ }^{4}$ It is noted in $[7$, p. 31] that termination of simply typed $\lambda$-calculus follows from termination of the Hyland-Wadsworth-labelling, a variation of which we use to formalize FFD.
} 
It follows directly from the definition that $\mathrm{h}(M) \neq \emptyset$, for all simply typed $\lambda$-terms $M$. Additionally, we show that the map h commutes over substitution:

Lemma A.1. $\mathrm{h}(M[x \mapsto N])=\mathrm{h}(M)[x \mapsto \mathrm{h}(N)]$.

Proof. By induction on $M$.

We have the following correspondence between the simply typed $\lambda$-calculus and $\mathcal{L} a m^{\rightarrow}$.

Lemma A.2. If $M \rightarrow_{\beta} N$ and $s \in \mathrm{h}(M)$, then there is a $t \in \mathrm{h}(N)$ such that $s \rightarrow \mathcal{L} a m \rightarrow t$.

Proof. By induction on the derivation of $M \rightarrow_{\beta} N$. The interesting case is if the $\beta$-step occurs at the head, i.e.:

$$
M=\left(\lambda x_{\alpha} \cdot M_{1}\right) M_{2} \quad \text { and } \quad N=M_{1}\left[x \mapsto M_{2}\right]
$$

By definition, this means that $s=\mathrm{T}_{\beta}^{n}\left(\operatorname{app}\left(\mathrm{T}_{\alpha \rightarrow \beta}^{m}\left(\operatorname{lam}\left(\lambda x \cdot t_{1}\right)\right), t_{2}\right)\right)$, where $t_{i} \in$ $\mathrm{h}\left(M_{i}\right)$, for $i \in\{1,2\}$, and $m, n>0$. Now:

$$
s \rightarrow \mathrm{T}_{\beta}^{n+1}\left(t_{1}\left[x \mapsto \mathrm{T}_{\alpha}\left(t_{2}\right)\right]\right) \in \mathrm{h}(N)
$$

where the last inclusion follows from Lemma A.1 and the facts that $\mathrm{T}_{\beta}^{n+1} \in$ $\mathrm{h}\left(M_{1}\right)$ because $t_{1} \in \mathrm{h}\left(M_{1}\right)$, and $\mathrm{T}_{\alpha}\left(t_{2}\right) \in \mathrm{h}\left(M_{2}\right)$ because $t_{2} \in \mathrm{h}\left(M_{2}\right)$.

Termination of $\mathcal{L} a m \rightarrow$ cannot be proved by any higher-order termination technique that I know of. However, it does follow from Finite Family Developments:

Proposition A.3. $\mathcal{L} a m \rightarrow$ is terminating.

Proof. Note that $\mathcal{L} a m \rightarrow$ is non-collapsing, and consider the $\omega$-labelling of $\mathcal{L} a m \rightarrow$, which consists of rules of the form:

$$
\operatorname{app}^{\ell}\left(\left(\left(\mathrm{T}_{\alpha \rightarrow \beta}^{k}\right)^{n}\left(\operatorname{lam}^{j}(\lambda x . Z(x))\right), Y\right) \rightarrow \mathrm{T}_{\beta}^{p}\left(Z\left(\mathrm{~T}_{\alpha}^{p}(Y)\right)\right)\right.
$$

where $\ell, j$ are labels representing the creation depth of the symbols, $\bar{k}$ is a sequence of $n$ such labels and $p=\max (\ell, \bar{k}, j)$. The symbols of the form $\mathrm{T}_{\alpha}$ will be called type symbols.

Let the height of a type be defined as follows: $h t(a)=1$ (where $a$ is a base type); $h t(\alpha \rightarrow \beta)=\max (h t(\alpha), h t(\beta))+1$. Now we can define the value of a type symbol as $\operatorname{Val}\left(\mathrm{T}_{\alpha}^{\ell}\right)=\ell+h t(\alpha)$ and the value of a term as $\operatorname{Val}(s)=\max \{\operatorname{Val}(f) \mid$ $f \in \operatorname{Sym}(s)\}$. Now, a simple induction on the context of the step reviels that, if $s \rightarrow \mathcal{L} a m \rightarrow t$, then $\operatorname{Val}(s) \geq \operatorname{Val}(t)$.

Let $\mathcal{R}: s_{0} \rightarrow s_{1} \rightarrow s_{2} \rightarrow \cdots$ be an arbitrary $\mathcal{L} a m \rightarrow$-reduction. Because $\operatorname{Val}\left(s_{i}\right) \geq \operatorname{Val}\left(s_{j}\right)$ if $i<j$, the value of every term in $\mathcal{R}$ is less or equal to $\operatorname{Val}\left(s_{0}\right)$, and thus every symbol occurring in the reduction has a creation depth less than or equal to $\operatorname{Val}\left(s_{0}\right)$. Therefore, by Finite Family Developments (Theorem 5.2), $\mathcal{R}$ is finite. 


\section{References}

1. Thomas Arts and Jürgen Giesl. Termination of term rewriting using dependency pairs. Theoretical Computer Science, 236(1-2):133-178, 2000.

2. Inge Bethke, Jan Willem Klop, and Roel de Vrijer. Descendants and origins in term rewriting. Information and Computation, 159(1-2):59-124, 2000.

3. Roel Bloo. Preservation of Termination for Explicit Substitution. PhD thesis, Technische Universiteit Eindhoven, 1997.

4. H. J. Sander Bruggink. A proof of finite family developments for higher-order rewriting using a prefix property. In RTA, 2006.

5. Gilles Dowek, Thérèse Hardin, and Claude Kirchner. Higher order unification via explicit substitutions. Information and Computation, 157(1-2):184-233, 2000.

6. J.M.E. Hyland. A syntactic characterization of the equality in some models of the $\lambda$-calculus. Journal of the London Mathematical Society, 12(2):361-370, 1976.

7. J. W. Klop. Combinatory Reduction Systems. PhD thesis, Utrecht Univ., 1980.

8. Jean-Jacques Lévy. Réductions correctes et optimales dans le $\lambda$-calcus. PhD thesis, Université Paris VII, 1978.

9. Luc Maranget. Optimal derivations in weak lambda-calculi and in orthogonal term rewriting systems. In $P O P L, 1991$.

10. Richard Mayr and Tobias Nipkow. Higher-order rewrite systems and their confluence. Theoretical Computer Science, 192:3-29, 1998.

11. Dale Miller. A logic programming language with lambda abstraction, function variables and simple unification. Journal of Logic and Computation, 1(4), 1991.

12. Tobias Nipkow. Higher-order critical pairs. In LICS, 1991.

13. W. W. Tait. Intensional interpretation of functionals of finite type I. Journal of Symbolic Logic, 32:198-212, 1967.

14. Terese. Term Rewriting Systems. Number 55 in CTTCS. CUP, 2003.

15. D.T. van Daalen. The language theory of Automath. $\mathrm{PhD}$ thesis, Technische Universiteit Eindhoven, 1980.

16. Vincent van Oostrom. Confluence for Abstract and Higher-Order Rewriting. PhD thesis, Vrije Universiteit Amsterdam, 1994.

17. Vincent van Oostrom. Finite family developments. In RTA, 1997.

18. Vincent van Oostrom and Roel de Vrijer. Four equivalent equivalences of reductions. ENTCS, 70(6), 2002.

19. C. P. Wadsworth. The relation between computational and denotational properties for Scott's $D_{\infty}$-models of the $\lambda$-calculus. SIAM Journal on Computing, 5, 1976. 